\title{
Long-time asymptotics for a 1D nonlocal porous medium equation with absorption or convection
}

DOI:

10.1142/S0219199719500159

\section{Document Version}

Accepted author manuscript

Link to publication record in Manchester Research Explorer

\section{Citation for published version (APA):}

Feo, F., Huang, Y., \& Volzone, B. (2019). Long-time asymptotics for a 1D nonlocal porous medium equation with absorption or convection. Communications in Contemporary Mathematics.

https://doi.org/10.1142/S0219199719500159

\section{Published in:}

Communications in Contemporary Mathematics

\section{Citing this paper}

Please note that where the full-text provided on Manchester Research Explorer is the Author Accepted Manuscript or Proof version this may differ from the final Published version. If citing, it is advised that you check and use the publisher's definitive version.

\section{General rights}

Copyright and moral rights for the publications made accessible in the Research Explorer are retained by the authors and/or other copyright owners and it is a condition of accessing publications that users recognise and abide by the legal requirements associated with these rights.

\section{Takedown policy}

If you believe that this document breaches copyright please refer to the University of Manchester's Takedown Procedures [http://man.ac.uk/04Y6Bo] or contact uml.scholarlycommunications@manchester.ac.uk providing relevant details, so we can investigate your claim.

\section{OPEN ACCESS}




\title{
Long-time asymptotics for a 1D nonlocal porous medium equation with absorption or convection
}

\author{
Filomena Feo * $\quad$ Yanghong Huang ${ }^{\dagger} \quad$ Bruno Volzone *
}

December 18, 2018

\begin{abstract}
In this paper, the long-time asymptotic behaviours of one-dimensional porous medium equations with a fractional pressure and absorption or convection are studied. In the parameter regimes when the nonlocal diffusion is dominant, the entropy method is adapted to derive the exponential convergence of relative entropy of solutions in similarity variables.
\end{abstract}

\section{Introduction}

A large variety of models for conserved quantities in continuum mechanics or physics are described by the continuity equation $u_{\tau}+\nabla \cdot(u \mathbf{v})=0$, where the density distribution $u(y, \tau)$ evolves in time $\tau$ following a velocity field $\mathbf{v}(y, \tau)$. According to Darcy's law, the velocity $\mathbf{v}$ is usually derived from a potential $p$ in the form $\mathbf{v}=-\mathcal{D} \nabla p$ for some tensor $\mathcal{D}$. In the porous media, the power-law relation $p=\frac{m}{m-1} u^{m-1}$ for $m>1$ is commonly proposed, leading to one of the canonical nonlinear diffusion equations reviewed in the monograph [40. Although this kind of local constitutive relations were successful in numerous practical models, there are situations where the potential (or pressure) $p$ depends non-locally on the density distribution $u$ [21]. The simplest prototypical example is $p=(-\Delta)^{-s} u$ with $s<N / 2$, expressed as the Riesz potential of $u$, i.e.,

$$
p(y, t)=(-\Delta)^{-s} u(y, t)=C_{N, s} \int_{\mathbb{R}^{N}}|y-z|^{2 s-N} u(z, t) \mathrm{d} z
$$

where the constant $C_{N, s}=\pi^{-N / 2} 2^{-s} \Gamma(N / 2-s) / \Gamma(s)$ is written in terms of the Euler Gamma function $\Gamma(z)$. The resulting evolution equation then becomes

$$
u_{\tau}-\nabla \cdot\left(u \nabla(-\Delta)^{-s} u\right)=0
$$

which can be defined for all $s \in(0,1)$. Basic questions like existence, uniqueness and regularity of solutions have been studied thoroughly in [9, 10], followed by generalizations to other related models in [3, 38]. While in general it is difficult to obtain quantitative properties of solutions to non-local nonlinear equations, Eq. (2) possesses special features that enable one to study the long term behaviours in terms of its selfsimilar solution. The self-similar profile, also called Barenblatt profile, was initially characterized by an obstacle problem [9], and was then explicitly constructed in [3]. Using similarity variables motivated from the scaling relations, the transformed equation has an entropy function so that the convergence towards the self-similar profile in one dimension can be established by the well-known entropy method in [11].

\footnotetext{
*Dipartimento di Ingegneria, Università degli Studi di Napoli "Pathenope", Centro Direzionale Isola C4 80143 Napoli, Italy. E-mail: filomena.feo@uniparthenope.it; bruno.volzone@uniparthenope.it

${ }^{\dagger}$ School of Mathematics, The University of Manchester, Oxford Road, Manchester, M13 9PL, United Kingdom. Email: yanghong.huang@manchester.ac.uk
} 
In this paper, we consider two variants of the porous medium equation (2) with nonlocal pressure. If the medium is lossy, the continuous density is dissolved, leading to the porous medium equation with absorption

$$
u_{\tau}-\nabla \cdot\left(u \nabla(-\Delta)^{-s} u\right)=-u^{r} .
$$

On the other hand, if the density moves with a density dependent local velocity, the equation with convection becomes

$$
u_{\tau}-\nabla \cdot\left(u \nabla(-\Delta)^{-s} u\right)=-\mathbf{b} \cdot \nabla u^{q},
$$

for some constant vector $\mathbf{b} \in \mathbb{R}^{N}$. In general, when dealing with solutions of both signs, the right-hand side of (3) or (4) should be replaced by $|u|^{r-1} u$ and $-\mathbf{b} \cdot \nabla\left(|u|^{q-1} u\right)$ respectively. However, if the initial data are non-negative as we assume below, the solutions are also non-negative on the time interval of existence, by following the arguments of [10, 3].

For the classical heat equation or porous medium equation, such variants with absorption [24, 26, 22, 32 or convection [23, 28, 33] have been studied intensively in the past, where the long term asymptotic behaviours depend on the interplay between the diffusion and absorption/convection, usually dictated by appropriate scaling transforms. However, for Eq. (3) or (4) of our interests here, the presence of nonlinear nonlocal diffusion makes refined techniques such the comparison principle no longer valid, presenting great challenges in the investigation of quantitative properties of the resulting solutions.

In this paper, we will focus on the long time behaviours of solutions to (3) and (4) mainly in the regime when the nonlocal nonlinear diffusion is dominant $(r>4-2 s$ and $q>3-2 s$ respectively), such that the absorption or convection essentially becomes perturbations added to Eq. (2). We remark that the absence of a comparison principle for the fractional potential pressure equation (2) (see e.g. [10] and the forthcoming paper [18]) forces in discarding the classical approach used in the proof of the convergence to the equilibrium in the local case, mainly based on scaling-compactness arguments and a suitable employment of the comparison principle itself (see e.g [26], [23, [27]). Thus the main technique is to extend the entropy method that was essential in establishing the precise convergence rate of Eq. (2) in [11. The entropy method, originally developed in the study of long term behaviour of solutions to the Fokker-Planck equation [2, 39], has been developed into a powerful framework to study the convergence of solutions towards their equilibrium. The key step is to relate the entropy dissipation rate with the relative entropy with different approaches, like convex Sobolev inequalities [1] or the second order derivative of the entropy [12. As a result, in order to prove the precise convergence rate of Eq. (2) to the equilibrium, the entropy method in [11] will be adapted in our context. Although the basic setting as in [11] is used here, several key observations have been made to recover the near optimal convergence rate with the presence of additional absorption or convection terms. When computing the evolution of the relative entropy along the flow, the main difficulty arises in finding reasonable sharp upper bounds of these terms by means of the relative entropy itself. Furthermore, a particular care is devoted in the approximation procedure in the general (nonsmooth) case, requiring extra regularity information of the minimizers of the relative entropies associated to the approximating problems.

To proceed, basic estimates for solutions of Eq. (3) and (4) will be reviewed first in Section 2, and the main results about the convergence of solutions will be stated. In Section 3, the exponential convergence of the relative entropy between the solutions to Eq. (3) and their time-dependent Barenblatt profiles are proved, followed by the proof of similar convergence rates for Eq. (4) with convection in Section 4. We conclude this paper in Section 5 with generalizations to related equations and other open problems.

\section{Basic estimates and main results}

Before discussing long-term asymptotic behaviours of Eq. (3) or (4), we first review a few fundamental questions about the existence and uniqueness of the solutions in appropriate spaces. To treat initial data as wide as possible, the usual notion of weak solutions using test functions can be introduced, as in [10, 7, 3, 36] for related equations, provided that the extra terms corresponding to absorption or 
convection are well-defined. The proof of the existence such weak solutions is now standard, mainly by taking the limit of regularized equations, by adding linear diffusion, removing the singularity in the nonlocal operator and confining the solution on bounded domains (see [10, 36] for more details). Here for simplicity, we only focus on the simplest yet the most important regularization technique with linear diffusion, and define the weak solutions as the limit of solutions to the regularized problems

$$
\frac{\partial u^{\epsilon}}{\partial \tau}-\nabla \cdot\left(u^{\epsilon} \nabla(-\Delta)^{-s} u^{\epsilon}\right)=-\left(u^{\epsilon}\right)^{r}+\epsilon \Delta u^{\epsilon}
$$

to Eq. (3) or

$$
\frac{\partial u^{\epsilon}}{\partial \tau}-\nabla \cdot\left(u^{\epsilon} \nabla(-\Delta)^{-s} u^{\epsilon}\right)=-\mathbf{b} \cdot \nabla\left(u^{\epsilon}\right)^{q}+\epsilon \Delta u^{\epsilon},
$$

to Eq. (4), without resorting to more complicated functional theoretic settings or approximation methods. The convergence of the regularized solutions $u^{\epsilon}$ to the weak solutions of Eq. (3) or (4) is only assumed to be weak* for non-negative measures, so that the main results are still valid, independent of the particular type of convergence for the limiting solution sequences in other (more regular) spaces. However, the question about the uniqueness of these weak solutions remains open, except in some special cases in one dimension [4], or in the context of more general types of equations for the integrated version [16]. To focus on the asymptotic behaviours of our interests here, we shall deal with the weak solutions obtained by taking limits from (5) or (6). Before showing basic estimates of the solutions, we first need several inequalities related to the nonlocal operator, in standard $L^{p}\left(\mathbb{R}^{N}\right)$ space with the norm $\|f\|_{p}=\left(\int_{\mathbb{R}^{N}}|f|^{p}\right)^{1 / p}$. These inequalities are valid for functions in larger spaces by density arguments. Whenever possible, the dependence of the constants on other parameters is specified and could be different from one line to another in the derivation of the estimates later. The first inequality is related to the Riesz potential operator $(-\Delta)^{-s}$ defined in (1), or equivalently as a multiplier $|\xi|^{-\alpha}$ in the Fourier space, i.e., $\mathcal{F}\left[(-\Delta)^{-\alpha / 2} f\right](\xi)=|\xi|^{-\alpha} \mathcal{F}[f](\xi)$.

Lemma 2.1 (Hardy-Littlewood-Sobolev inequality). Let $0<s<N$ and $1<p<q<\infty$ with the relation $1 / q=1 / p-s / N$. If $f \in L^{p}\left(\mathbb{R}^{N}\right)$, then $(-\Delta)^{-s / 2} f \in L^{q}\left(\mathbb{R}^{N}\right)$. Moreover, there is a constant $C(N, s, p)$ such that

$$
\left\|(-\Delta)^{-s / 2} f\right\|_{q} \leq C(N, s, p)\|f\|_{p}
$$

Associated with the Riesz operator is its inverse, the fractional Laplacian $(-\Delta)^{\alpha / 2}$, as a multiplier $|\xi|^{\alpha}$ in the Fourier space, i.e, $\mathcal{F}\left[(-\Delta)^{\alpha / 2} f\right](\xi)=|\xi|^{\alpha} \mathcal{F}[f](\xi)$. The natural space related to the fractional Laplacian is $\dot{H}^{\alpha / 2}\left(\mathbb{R}^{N}\right)$, the set of all functions $f$ such that

$$
\|f\|_{\dot{H}^{\alpha / 2}}:=\left\|(-\Delta)^{\alpha / 2} f\right\|_{2}=\left(\int_{\mathbb{R}^{N}}|\xi|^{\alpha}|\mathcal{F}[f](\xi)|^{2} \mathrm{~d} \xi\right)^{1 / 2}<\infty .
$$

Although one can not manipulate identities about functions with nonlocal operators in the same ways as for classical derivatives, there are still powerful inequalities like the following ones that are crucial in the proof of basic estimates below.

Lemma 2.2 (Stroock-Varopoulos inequality). For $p \geq 1, \alpha \in(0,2]$, and any non-negative function $w \in C_{c}^{\infty}\left(\mathbb{R}^{N}\right)$, the following inequality holds true

$$
\int w^{p}(-\Delta)^{\frac{\alpha}{2}} w \mathrm{~d} x \geq \frac{4 p}{(p+1)^{2}} \int\left|(-\Delta)^{\frac{\alpha}{4}} w^{\frac{p+1}{2}}\right|^{2} \mathrm{~d} x=\frac{4 p}{(p+1)^{2}} \int w^{\frac{p+1}{2}}(-\Delta)^{\frac{\alpha}{2}} w^{\frac{p+1}{2}} \mathrm{~d} x .
$$

Lemma 2.3 (Fractional Gagliardo-Nirenberg inequality). 1) Let $0<\alpha \leq 2$. If $u$ is a non-negative function such that $u \in L^{1}\left(\mathbb{R}^{N}\right)$ and $u^{m / 2} \in \dot{H}^{\alpha / 2}\left(\mathbb{R}^{N}\right)$ for some $m>2$, then $u \in L^{l}\left(\mathbb{R}^{N}\right)$ for any $l \in(m / 2, m)$ and

$$
\|u\|_{l}^{a} \leq C_{N, \alpha}\left\|(-\Delta)^{\alpha / 4} u^{m / 2}\right\|_{2}^{2}\|u\|_{1}^{b}
$$


where $C_{N, \alpha}$ is a constant depending only on $N$ and $\alpha$,

$$
a=\frac{l(\alpha+N(m-1))}{N(l-1)}, \quad b=a-m=\frac{\alpha l+N(m-l)}{N(l-1)} .
$$

2) Let $l \geq 1,0<\alpha<\min \{N, 2\}$. Then for any $v \in L^{l}\left(\mathbb{R}^{N}\right) \cap \dot{H}^{\alpha}\left(\mathbb{R}^{N}\right)$,

$$
\|v\|_{m}^{\theta+1} \leq C_{1}\left\|(-\Delta)^{\frac{\alpha}{2}} v\right\|_{2}\|v\|_{l}^{\theta}
$$

where $m=\frac{N(l+2)}{2(N-\alpha)}, \theta=\frac{l}{2}$ and the constant $C_{1}=C(N, \alpha) \frac{(l+2)^{2}}{8(l+1)}$.

The proof of the Strook-Varopoulos inequality can be found in [29], and for the proof of (9) or (10), see [3, Lemma 3.2], [17, Lemma 5.3], [20, Proposition 2.1] or the survey [19]. With all the preliminary inequalities, we can derive the basic estimates for solutions to (3) or (4).

Proposition 2.4 (Basic estimates for equation with absorption). Let $u$ be a weak solution to Eq. (3) obtained as the limit of solutions $u^{\epsilon}$ to Eq. (5) with non-negative initial data $u^{\epsilon}(0)=u_{0}, r>1$ and $1 \leq p \leq \infty$. If $u_{0} \in L^{p}\left(\mathbb{R}^{N}\right) \cap L^{1}\left(\mathbb{R}^{N}\right)$, we have

$$
\|u(\tau)\|_{p} \leq\left\|u_{0}\right\|_{p}, \quad \tau \geq 0 .
$$

Moreover if $u_{0} \in L^{1}\left(\mathbb{R}^{N}\right)$ the following estimate

$$
\|u(\tau)\|_{p} \leq C\left(\left\|u_{0}\right\|_{1}, N, p, r, s\right) \tau^{-\max \left(\frac{N(p-1)}{p(N+2-2 s)}, \frac{p-1}{p(r-1)}\right)}, \quad \tau>0,
$$

holds for a positive constant $C\left(\left\|u_{0}\right\|_{1}, N, p, r, s\right)$ depending on $N, p, r, s$ and on the initial condition only through the total mass $\left\|u_{0}\right\|_{1}$.

Proof. The non-negativity of solutions $u^{\epsilon}(\tau)$ can be proved similarly as in [10, 3, and is skipped for simplicity. Then the fact $\frac{\mathrm{d}}{\mathrm{d} \tau}\left\|u^{\epsilon}(\tau)\right\|_{1}=-\int\left(u^{\epsilon}(\tau)\right)^{r} \mathrm{~d} x \leq 0$ immediately implies that $\left\|u^{\epsilon}(\tau)\right\|_{1} \leq\left\|u^{\epsilon}(0)\right\|_{1}=$ $\left\|u_{0}\right\|_{1}$. Similarly, for any finite $p>1$,

$$
\begin{aligned}
\frac{1}{p} \frac{\mathrm{d}}{\mathrm{d} \tau}\left\|u^{\epsilon}(\tau)\right\|_{p}^{p} & =\epsilon \int\left(u^{\epsilon}\right)^{p-1} \Delta u^{\epsilon} \mathrm{d} x+\int \nabla \cdot\left(u^{\epsilon} \nabla(-\Delta)^{-s} u^{\epsilon}\right)\left(u^{\epsilon}\right)^{p-1} \mathrm{~d} x-\int\left(u^{\epsilon}\right)^{r+p-1} \mathrm{~d} x \\
& =-(p-1) \epsilon \int\left(u^{\epsilon}\right)^{p-2}\left|\nabla u^{\epsilon}\right|^{2} \mathrm{~d}-\frac{p-1}{p} \int\left(u^{\epsilon}\right)^{p}(-\Delta)^{1-s} u^{\epsilon}-\int\left(u^{\epsilon}\right)^{r+p-1} \mathrm{~d} x \\
& \leq-\frac{4(p-1)}{(p+1)^{2}} \int\left|(-\Delta)^{\frac{1-s}{2}}\left(u^{\epsilon}\right)^{\frac{p+1}{2}}\right|^{2} \mathrm{~d} x-\int\left(u^{\epsilon}\right)^{r+p-1} \mathrm{~d} x,
\end{aligned}
$$

where the Stroock-Varopoulos inequality (8) is used in the last step. Since all terms in the last line of (13) are non-positive, we can conclude that

$$
\left\|u^{\epsilon}(\tau)\right\|_{p} \leq\left\|u^{\epsilon}(0)\right\|_{p}=\left\|u_{0}\right\|_{p} \quad \text { for } \tau \geq 0 .
$$

If $u_{0} \in L^{\infty}\left(\mathbb{R}^{N}\right) \cap L^{1}\left(\mathbb{R}^{N}\right)$, taking the limit as $p \rightarrow+\infty$ in 14 the following estimate holds

$$
\left\|u^{\epsilon}(\tau)\right\|_{\infty} \leq\left\|u_{0}\right\|_{\infty} \quad \text { for } \tau \geq 0 .
$$

To obtain refined decay rate along the evolution, the last two terms in (13) can be related to $\left\|u_{\epsilon}(\tau)\right\|_{p}$ to establish a self-contained differential inequality. By choosing $l=p, \alpha=2-2 s$ and $m=p+1$, the fractional Gagliardo-Nirenberg inequality (9) becomes

$$
\left\|u^{\epsilon}\right\|_{p}^{a} \leq C_{N, \alpha}\left\|(-\Delta)^{\frac{1-s}{2}}\left(u^{\epsilon}\right)^{\frac{p+1}{2}}\right\|_{2}^{2}\left\|u^{\epsilon}\right\|_{1}^{b} \leq C_{N, \alpha}\left\|(-\Delta)^{\frac{1-s}{2}}\left(u^{\epsilon}\right)^{\frac{p+1}{2}}\right\|_{2}^{2}\left\|u_{0}\right\|_{1}^{b}
$$


with $a=\frac{p(N p+2-2 s)}{N(p-1)}$ and $b=\frac{N+2 p-2 s p}{N(p-1)}$. On the other hand, we have the Hölder inequality

$$
\left\|u^{\epsilon}\right\|_{p} \leq\left\|u^{\epsilon}\right\|_{r+p-1}^{\frac{(r+p-1)(p-1)}{(r+p-2) p}}\left\|u^{\epsilon}\right\|_{1}^{\frac{(r-1)}{p(r+p-2)}} \leq\left\|u^{\epsilon}\right\|_{r+p-1}^{\frac{(r+p-1)(p-1)}{(r+p-2) p}}\left\|u_{0}\right\|_{1}^{\frac{(r-1)}{p(r+p-2)}}
$$

Therefore, the differential inequality (13) suggests the following two decay rates of $\left\|u^{\epsilon}(\tau)\right\|_{p}$,

$$
\frac{1}{p} \frac{\mathrm{d}}{\mathrm{d} \tau}\left\|u^{\epsilon}(\tau)\right\|_{p}^{p} \leq-\frac{4(p-1)}{C_{N, \alpha}(p+1)^{2}\left\|u_{0}\right\|_{1}^{b}}\left\|u^{\epsilon}(\tau)\right\|_{p}^{a} \quad \text { and } \quad \frac{1}{p} \frac{\mathrm{d}}{\mathrm{d} \tau}\left\|u^{\epsilon}(\tau)\right\|_{p}^{p} \leq-\frac{\left\|u^{\epsilon}\right\|_{p}^{\frac{p(r+p-2)}{p-1}}}{\left\|u_{0}\right\|_{1}^{\frac{r-1}{p-1}}},
$$

from which we get

$$
\left\|u^{\epsilon}(\tau)\right\|_{p} \leq\left(\frac{4 p(N+2-2 s)}{N C_{N, \alpha}(p+1)^{2}\left\|u_{0}\right\|_{1}^{b}} \tau\right)^{-\frac{N(p-1)}{p(N+2-2 s)}} \quad \text { and } \quad\left\|u^{\epsilon}(\tau)\right\|_{p} \leq\left(\frac{p(r-1)}{p-1}\left\|u_{0}\right\|_{1}^{-\frac{r-1}{p-1}} \tau\right)^{-\frac{p-1}{p(r-1)}} .
$$

These two bounds lead to the estimate $\left\|u^{\epsilon}(\tau)\right\|_{p} \leq C\left(\left\|u_{0}\right\|_{1}, N, p, r, s\right) \tau^{-\max \left(\frac{N(p-1)}{p(N+2-2)}, \frac{p-1}{p(r-1)}\right)}$, where the constant depends only on certain powers of $\left\|u_{0}\right\|_{1}$. The desired estimates (11) and (12) then hold by taking the limit as $\epsilon$ goes to zero.

However, the case for $p=\infty$ in $(12)$ can not be obtained directly by taking the limit as $p$ goes to infinity, because the constant $C\left(\left\|u_{0}\right\|_{1}, N, p, r, s\right)$ becomes infinity. Instead, the proof in [3, Theorem 6.1] for $L^{1}-L^{\infty}$ smoothing effect of solutions is adapted.

Remark 2.5. The two decay rates in (12) actually correspond to two different regimes for the behaviours of the solutions: in the diffusion dominated regimes with $r>(2 N+2-2 s) / N$, the decay rate $\tau^{-N(p-1) / p(N+2-2 s)}$ prevails and the constant $C$ does not depend on $r$; otherwise the diffusion and the absorption balance each other, and the rate $\tau^{-(p-1) / p(r-1)}$ prevails. We will mainly focus on the first case, where the entropy method can be applied in the transformed equation with similarity variables.

For Eq. (4) with convection, similar computation can be applied to obtain the following estimates.

Proposition 2.6 (Basic estimates for equation with convection). Let $u$ be a weak solution to Eq. (4) obtained as the limit of solutions $u^{\epsilon}$ to Eq. (6) with non-negative initial data $u^{\epsilon}(0)=u_{0}, q>1$ and $1 \leq p \leq \infty$. If $u_{0} \in L^{1}\left(\mathbb{R}^{N}\right) \cap L^{p}\left(\mathbb{R}^{N}\right)$, we have

$$
\|u(\tau)\|_{p} \leq\left\|u_{0}\right\|_{p}, \quad \tau \geq 0 .
$$

Moreover if $u_{0} \in L^{1}\left(\mathbb{R}^{N}\right)$ the following inequality

$$
\|u(\tau)\|_{p} \leq C\left(\left\|u_{0}\right\|_{1}, N, p, q, s\right) \tau^{-\frac{N(p-1)}{p(N+2-2 s)}}, \quad \tau>0,
$$

holds for a positive constant $C\left(\left\|u_{0}\right\|_{1}, N, p, q, s\right)$ depending on $N, s, p$ and on the initial total mass $\left\|u_{0}\right\|_{1}$.

In this paper, we concentrate on cases where the absorption in Eq. (3) or the convection in Eq. (4) is dominated by the nonlocal diffusion, so that the long term asymptotic behaviours are essentially governed by Eq. (2). As a result, the same change of similarity variables used to study Eq. (2) in [9, 3, 11] is adopted here, that is,

$$
x=y(1+\lambda \tau)^{-1 / \lambda}, \quad t=\frac{1}{\lambda} \log (1+\lambda \tau), \quad \rho(x, t)=(1+\lambda \tau)^{N / \lambda} u(y, \tau)
$$

with $\lambda=N+2-2 s$. In this way, the absorption or convection becomes exponentially small perturbations as shown in the next two sections. To better illustrate the main techniques involved, we first review the convergence of the transformed equation

$$
\rho_{t}-\nabla_{x} \cdot\left(\rho \nabla_{x}(-\Delta)^{-s} \rho+x \rho\right)=0,
$$


obtained from Eq. (2) via the similar variables (15). It is show in [9] that, the solution $\rho(x, t)$ converges to a steady state, which can be characterized as the solution of a fractional obstacle problem. The steady state $\rho_{M}(x)$, depending on the total conserved mass $M=\int \rho(x, 0) \mathrm{d} x=\int u(x, 0) \mathrm{d} x$, is given explicitly in [3] as

$$
\rho_{M}(x)=\frac{2^{2 s-1} \Gamma(1+N / 2)}{\Gamma(2-s) \Gamma(1-s+N / 2)}\left(R^{2}-|x|^{2}\right)_{+}^{1-s},
$$

where the radius of support $R$ is determined by the total conserved mass $M$ through the relation

$$
M=\int \rho_{M}(x) \mathrm{d} x=\frac{2^{2 s} \pi^{N / 2} \Gamma(1+N / 2)}{(N+2-2 s) \Gamma(1-s+N / 2)^{2}} R^{N+2-2 s} .
$$

Because of the presence both nonlinearity and nonlocality, the convergence of the solution $\rho(x, t)$ towards $\rho_{M}(x)$ is more difficult, relying heavily on the Lyapunov function or the entropy

$$
H[\rho]=\int\left(\frac{1}{2} \rho(-\Delta)^{-s} \rho+\frac{1}{2}|x|^{2} \rho\right) \mathrm{d} x .
$$

Since $H[\rho]$ is a convex functional, it is proved in [15, Theorem 1.1] that the steady state (17) is the unique minimizer of $H[\rho]$ on the space of all non-negative measures with total mass $M$ and is characterized by the relation

$$
(-\Delta)^{-s} \rho_{M}(x)+\frac{1}{2}|x|^{2}=\frac{N}{2(N-2 s)} R^{2}, \quad|x| \leq R,
$$

and

$$
(-\Delta)^{-s} \rho_{M}(x)+\frac{1}{2}|x|^{2} \geq \frac{N}{2(N-2 s)} R^{2}, \quad|x|>R .
$$

However, more refined questions like the convergence rate of $\rho(x, t)$ towards $\rho_{M}(x)$ seem less likely to be answered in the framework of this classical notion of convexity. Instead, it is more convenient to study the displacement convexity originated by McCann [30, a key concept in the theory of optimal transport [42, 35]: by defining the entropy dissipation rate

$$
I[\rho]=\int \rho\left|\nabla(-\Delta)^{-s} \rho+x\right|^{2} \mathrm{~d} x
$$

which is exactly $-\frac{d}{d t} H[\rho]$ when $\rho$ is governed by $(16)$, the main task is to establish a relationship between the relative entropy $H\left[\rho \mid \rho_{M}\right]:=H[\rho]-H\left[\rho_{M}\right]$ and the entropy dissipation rate $I[\rho]$. Such a relationship, also called entropy-entropy dissipation inequality, lies at the heart of the entropy method in proving convergence and is established in only one dimension in [11] as follows.

Lemma 2.7. Let $N=1, s<1 / 2$. If the entropy $H[\rho]$ and entropy dissipation rate $I[\rho]$ are defined as in (19) and (22) respectively, then the inequality

$$
H\left[\rho \mid \rho_{M}\right]:=H[\rho]-H\left[\rho_{M}\right] \leq \frac{1}{2} I[\rho]
$$

holds for any non-negative Radon measure $\rho$ with the same total mass as $\rho_{M}$ such that $H[\rho]$ is bounded.

Remark 2.8. It remains an open problem whether the inequality (23) still holds in higher dimension. Provided that it is satisfied, the main results in this paper can be generalized into higher dimensions. The constraint $s<1 / 2$ (and $s<N / 2$ in general dimension $N$ ) must be imposed, because of the appearance of terms like $\left\|(-\Delta)^{s / 2} \rho^{r}\right\|_{2}$ that have to be bounded by norms of $\rho$ using the Hardy-Littlewood-Sobolev inequality (as in Theorem 3.3). 
Formally, once a relationship like $(23)$ is established, the Gronwall type inequality

$$
\frac{\mathrm{d}}{\mathrm{d} t} H\left[\rho \mid \rho_{M}\right]=-I[\rho] \leq-2 H\left[\rho \mid \rho_{M}\right]
$$

implies the exponential convergence of the relative entropy, i.e. $H\left[\rho(t) \mid \rho_{M}\right] \leq e^{-2 t}\left(H\left[u_{0} \mid \rho_{M}\right]\right)$. Exponential convergence in other norms or metrics, for instance using Csiszár-Kullback inequality for norms of $\rho-\rho_{M}$ dominated by the relative entropy $H\left[\rho \mid \rho_{M}\right]$ are available in literature (see the monograph [25] for a detailed review). One such inequality in the present setting is also established in [11, Lemma 3.3], from which one can show the exponential convergence of the solution.

Lemma 2.9. Let $\rho$ be a non-negative function on $\mathbb{R}^{N}$ with the same total mass $M$ as the steady state $\rho_{M}$, then

$$
\left\|(-\Delta)^{-s / 2}\left(\rho-\rho_{M}\right)\right\|_{2}^{2} \leq 2\left(H\left[\rho \mid \rho_{M}\right]\right) .
$$

After all the basic estimates and background information about entropy methods, we now state the main theorems. They establish the asymptotic behaviours on the one-dimensional case, in terms of the relative entropy for the non transformed variables, and $H^{-s}(\mathbb{R})$ norm of the difference between the solution $u$ and the asymptotic profile

$$
u_{M}(y, \tau)=(1+\lambda \tau)^{-N / \lambda} \rho_{M}\left(y(1+\lambda \tau)^{-1 / \lambda}\right) .
$$

It is clear that (26) is the time-dependant Barenblatt profile for the fractional-pressure evolution equation (2).

Now we state the main results of the paper, namely the asymptotic convergence to the Barenblatt profile (26) for solutions to the models (3), (4) in terms of the relative entropy, in the diffusion dominated regime. To this aim, as pointed out in Section 2, we consider solutions obtained as limit of solutions $u_{\epsilon}$ to the approximating problems (5)-(6).

Theorem 2.10 (Asymptotic convergence for the equation with absorption). Let $N=1, u(y, \tau)$ be a weak solution of (3) obtained as a limit of solutions of (5) with non-negative initial data $u_{0} \in L^{1}(\mathbb{R},(1+$ $\left.\left.|x|^{2}\right) \mathrm{d} x\right) \cap L^{\infty}(\mathbb{R}), r>4-2 s$ and $s<1 / 2$. Let $u_{M(\tau)}$ be the rescaled profile 26) with the mass $M(\tau)=$ $\int u(y, \tau) d y$. Then there exists a constant $C$ depending on $r, s,\left\|u_{0}\right\|_{1},\left\|u_{0}\right\|_{\infty}$ and on $H\left[u_{0} \mid u_{M_{0}}\right]$ such that

$$
H\left[u(\tau) \mid u_{M(\tau)}\right] \leq C(1+|\log \tau|)^{2}(1+\lambda \tau)^{-\frac{1-2 s}{\lambda}-2 \min \left(\frac{1}{\lambda}, \delta\right)}
$$

with $\delta=(r-1) / \lambda-1>0$. In particular, we have

$$
\left\|(-\Delta)^{-s / 2}\left(u(\tau)-u_{M(\tau)}\right)\right\|_{2} \leq C(1+|\log \tau|)(1+\lambda \tau)^{-\frac{1-2 s}{2 \lambda}-\min \left(\frac{1}{\lambda}, \delta\right)} .
$$

Theorem 2.11 (Asymptotic convergence for the equation with convection). Let $N=1, u(y, \tau)$ be $a$ weak solution of (4) obtained as a limit of solutions of (6) with non-negative initial data $u_{0} \in L^{1}(\mathbb{R},(1+$ $\left.\left.|x|^{2}\right) \mathrm{d} x\right) \cap L^{\infty}(\mathbb{R}), q>3-2 s$ and $s<1 / 2$. Let $u_{M_{0}}$ be the rescaled profile $(26$ ) with the conserved mass $M_{0}=\left\|u_{0}\right\|_{1}$. Then there exists a constant $C$ depending on $q, s, M_{0},\left\|u_{0}\right\|_{\infty}$ and on $H\left[u_{0} \mid u_{M_{0}}\right]$ such that

$$
H\left[u(\tau) \mid u_{M_{0}}\right] \leq C(1+|\log \tau|)^{2}(1+\lambda \tau)^{-\frac{1-2 s}{\lambda}-2 \min \left(\frac{1}{\lambda}, \theta\right)}
$$

with $\theta=q / \lambda-1>0$. In particular we have

$$
\left\|(-\Delta)^{-s / 2}\left(u(\tau)-u_{M_{0}}\right)\right\|_{2} \leq C(1+|\log \tau|)(1+\lambda \tau)^{-\frac{1-2 s}{2 \lambda}-\min \left(\frac{1}{\lambda}, \theta\right)} .
$$

Remark 2.12. We remark that in the case treated in Theorem (2.11) we have the uniqueness of solutions (see Lemma 4.2).

The convergence of the solution $u(\tau)$ to $u_{M(\tau)}$ ( or $u_{M}$ ) in other metrics usually relies on interpolation inequalities between norms, and requires higher regularity on $u(\tau)-u_{M(\tau)}\left(\right.$ or $\left.u(\tau)-u_{M}\right)$ that is out of scope of this paper. This issue will be commented near the end of Subsection 3.4. 


\section{Fractional diffusion with absorption}

In this section, the long term asymptotic behaviours of non-negative solutions to the fractional diffusion equation (3) with absorption are studied. Different behaviours will be classified first based on the parameter $r$. The focus will on the regime when the absorption is dominated by the diffusion and can be ignored in the long run. Formal computations will be performed to illustrate the entropy method for smooth enough solutions and then for more general weak solutions by limiting procedure.

\subsection{Basic behaviours and the transformed equation in similarity variables}

Because the evolution equation (3) is governed by two forces, the fractional diffusion $\nabla \cdot\left(u \nabla(-\Delta)^{-s} u\right)$ and the absorption $-u^{r}$, the corresponding long term behaviours of the solutions are determined by the competition between these two effects, similar to the well studied cases where the nonlocal diffusion is replaced with classical linear or nonlinear diffusion [24, 31. Formally if the non-negative solution $u$ is smooth and uniformly bounded, $\nabla \cdot\left(u \nabla(-\Delta)^{-s} u\right)$ is non-positive at any global maximum of $u$ and $\|u(\tau)\|_{\infty}$ is dominated by the ODE $u_{\tau}=-u^{r}$. In other words, if $r \in(0,1)$, the solution vanishes in finite time, and if $r>1$, then

$$
\|u(\tau)\|_{\infty} \leq\left(\left\|u_{0}\right\|_{\infty}^{1-r}+(r-1) \tau\right)^{-1 /(r-1)} .
$$

Furthermore, if $r \geq 1$, the solution can not vanish in finite time. In fact, from the condition $\|u(t)\|_{\infty} \leq$ $\left\|u_{0}\right\|_{\infty}$ in Proposition 2.4, the change of the total mass of $u(\tau)$ satisfies the differential inequality

$$
\frac{\mathrm{d}}{\mathrm{d} \tau}\left(\int u(y, \tau) \mathrm{d} y\right)=-\int u(y, \tau)^{r} \mathrm{~d} y \geq-\left(\int u(y, \tau) \mathrm{d} y\right)\left\|u_{0}\right\|_{\infty}^{r-1} .
$$

The lower bound on the decreasing rate implies that the total mass can not be zero at any finite time.

The regime $r>1$ can be analysed further, depending on the role played by the nonlocal diffusion dictated by scaling argument. If $r \in(1,(2 N+2-2 s) / N)$, the long term behaviours are the determined by both the fractional diffusion and the absorption, and the solutions are expected to converge to the self-similar solution of the form

$$
(1+(r-1) \tau)^{-1 /(r-1)} U\left(y(1+(r-1) \tau)^{-\alpha /(r-1)}\right),
$$

where $\alpha=\frac{2-r}{2(1-s)}$ and the self-similar profile $U$ satisfies the equation

$$
U+\alpha x \cdot \nabla U+\nabla \cdot\left(U \nabla(-\Delta)^{-s} U\right)-U^{r}=0 .
$$

When $s=0$ (the case with classical diffusion), the convergence of the solutions towards the self-similar solutions can be established rigorously [24, 31, mainly using comparison principles. However, similar refined quantitative techniques do not seem to be available in the fractional setting; properties of the selfsimilar profile $U$ governed by $(29)$ and any further information about the convergence remain challenging open problems.

In this paper we are interested in the parameter regime when

$$
r>\frac{2 N+2-2 s}{N}
$$

such that the absorption becomes small perturbation in determining the long term behaviours. More precisely, using the same change of similarity variables (15), Eq. (3) becomes

$$
\rho_{t}=\nabla \cdot\left[\rho\left(\nabla(-\Delta)^{-s} \rho+x\right)\right]-P(t)^{-\delta} \rho^{r},
$$

where $P(t)=1+\lambda \tau=e^{\lambda t}$ and $\delta=N(r-1) / \lambda-1>0$. Similarly, basic estimates for $u(\tau)$ in Proposition 2.4 can be translated directly into those for $\rho(t)$. 
Proposition 3.1. Let the solution $\rho$ for Eq. (31) be obtained from the solution u for Eq. (3) using the change of similarity variables (15) and $1 \leq p \leq \infty$. Assume we are in diffusion-dominated regime (30). If $u_{0} \in L^{1}\left(\mathbb{R}^{N}\right) \cap L^{p}\left(\mathbb{R}^{N}\right)$, we have

$$
\|\rho(t)\|_{p} \leq\left\|u_{0}\right\|_{p} e^{N\left(1-\frac{1}{p}\right) t}, \quad t \geq 0 .
$$

Moreover if $u_{0} \in L^{1}\left(\mathbb{R}^{N}\right)$, the following estimates

$$
\begin{gathered}
\|\rho(t)\|_{p} \leq C\left(\left\|u_{0}\right\|_{1}, N, p, s\right), \quad \text { for } t>t_{0} \\
\|\rho(t)\|_{p} \leq C\left(\left\|u_{0}\right\|_{1}, N, p, s\right) t^{-\frac{N(p-1)}{p(N+2-2 s)}}, \quad \text { for } 0<t<t_{0}
\end{gathered}
$$

hold for some $t_{0}>0$.

Proof. The estimate $(32)$ is exactly $(11)$, in terms of the similarity variables $(15)$. In the parameter regime $r>(2 N+2-2 s) / N$, the estimate (12) becomes $\|u(\tau)\|_{p} \leq C\left(\left\|u_{0}\right\|_{1}, N, p, s\right) \tau^{-N(p-1) / p(N+2-2 s)}$ (with no dependence on $r$ in the constant $C$ ), or

$$
\|\rho(t)\|_{p} \leq\|u(\tau)\|_{p} e^{N\left(1-\frac{1}{p}\right) t} \leq C\left(\left\|u_{0}\right\|_{1}, N, p, s\right)\left(\frac{1-e^{-\lambda t}}{\lambda}\right)^{-\frac{N}{N+2-2 s}\left(1-\frac{1}{p}\right)} .
$$

Therefore the estimate (33) is obtained for $t>t_{0}$ and (34) for $0<t<t_{0}$, using the bounds $\left(1-e^{-\lambda t}\right) / \lambda \geq$ $\left(1-e^{-\lambda t_{0}}\right) / \lambda$ and $\left(1-e^{-\lambda t}\right) / \lambda \geq t\left(1-e^{-\lambda_{0} t}\right) /\left(\lambda t_{0}\right)$ respectively.

Remark 3.2. Using (32) and (33), if $u_{0} \in L^{1}\left(\mathbb{R}^{N}\right) \cap L^{p}\left(\mathbb{R}^{N}\right)$, then $\|\rho(t)\|_{p}$ is uniformly bounded for any time $t>0$ by a constant depending on $N, s, p,\left\|u_{0}\right\|_{1}$ and $\left\|u_{0}\right\|_{p}$. In fact, from (33), the uniform bound of $\left\|u_{0}\right\|_{p}$ on the initial condition $u_{0}$ is not needed for the long term behaviours of our interest. For simplicity, we will assume that $u_{0} \in L^{1}\left(\mathbb{R}^{N}\right) \cap L^{\infty}\left(\mathbb{R}^{N}\right)$ in the rest of the paper, thus we have

$$
\|\rho(t)\|_{\infty} \leq C\left(\left\|u_{0}\right\|_{1},\left\|u_{0}\right\|_{\infty}, N, s\right) .
$$

Consequently, by interpolation between norms, we also have $\|\rho(t)\|_{p} \leq C\left(\left\|u_{0}\right\|\left\|_{1},\right\| u_{0} \|_{\infty}, N, p, s\right)$ for any $1 \leq p \leq \infty$.

Let $M(t)=\int \rho(x, t) \mathrm{d} x$ be the total mass at time $t$. Since

$$
\frac{\mathrm{d}}{\mathrm{d} t} M(t)=-P(t)^{-\delta} \int \rho^{r} \mathrm{~d} x \leq 0,
$$

the mass $M(t)$ is decreasing, i.e. $\|\rho(t)\|_{1}:=M(t) \leq M_{0}:=\left\|u_{0}\right\|_{1}$. Moreover, we can show that the limiting mass

$$
M_{\infty}=\left\|u_{0}\right\|_{1}-\int_{0}^{+\infty} P(s)^{-\delta} \int \rho(x, s)^{r} \mathrm{~d} x \mathrm{~d} s
$$

is strictly positive. Indeed, from the uniform bound (35) of the solution, we get

$$
\frac{\mathrm{d}}{\mathrm{d} t} M(t)=-P(t)^{-\delta} \int \rho^{r} \mathrm{~d} x \geq-P(t)^{-\delta}\|\rho(t)\|_{\infty}^{r-1} \int \rho \mathrm{d} x \geq-C\left(\left\|u_{0}\right\|_{1},\left\|u_{0}\right\|_{\infty}, N, s\right) P(t)^{-\delta} M(t) .
$$

This differential inequality can be integrated on the time interval $(0, t)$ to obtain

$$
\log \left(\frac{M(t)}{M(0)}\right) \geq-C\left(\left\|u_{0}\right\|_{1},\left\|u_{0}\right\|_{\infty}, N, s\right) \int_{0}^{t} P(t)^{-\delta} \mathrm{d} t \geq-C\left(\left\|u_{0}\right\|_{1},\left\|u_{0}\right\|_{\infty}, N, s\right) \int_{0}^{\infty} P(t)^{-\delta} \mathrm{d} t .
$$

This implies that

$$
M(t) \geq M_{\infty} \geq M(0) \exp \left(-C\left(\left\|u_{0}\right\|_{1},\left\|u_{0}\right\|_{\infty}, N, s\right) \int_{0}^{\infty} P(t)^{-\delta} \mathrm{d} t\right)>0 .
$$

With this result, we can show that $\rho(x, t)$ eventually converges to the profile $\rho_{M_{\infty}}(x)$, by first showing that it converges to the time dependent profile $\rho_{M(t)}(x)$. 


\subsection{Exponential convergence for smooth solutions}

In this subsection, we show that if $\rho(t)$ is a solution to Eq. (31) with certain regularity, then it is "close" to the Barenblatt profile $\rho_{M(t)}$ measured in some metric. Here the main technique is to adapt the wellestablished entropy method in this new setting, by proving the exponential convergence of the relative entropy between the solution $\rho(t)$ and the Barenblatt profile $\rho_{M(t)}$. The calculation below is entirely formal, without worrying about the regularity of the solution $\rho(t)$, so that the main idea is conveyed in the simplest setting. Once this convergence is established, the convergence for general weak solutions through limiting procedure will be proved along the same limiting sequences, as shown in the next subsection.

The key idea is still to establish a self-contained differential equality for the relative entropy $H\left[\rho(t) \mid \rho_{M(t)}\right]$. From the definition of the entropy $H[\rho]$, if $\rho(t)$ is a solution of (31) and decays to zero fast enough, then the time change of the entropy becomes

$$
\frac{\mathrm{d}}{\mathrm{d} t} H[\rho(t)]=-I[\rho(t)]-P(t)^{-\delta} \int \rho(t)^{r}\left[(-\Delta)^{-s} \rho+\frac{x^{2}}{2}\right] \mathrm{d} x
$$

On the other hand, since the time-dependent profile $\rho_{M(t)}$ is supported only on the ball $|x| \leq R(t)$ with $R(t)$ related to the total mass $M(t)$ via 18 ,

$$
\begin{aligned}
\frac{\mathrm{d}}{\mathrm{d} t} H\left[\rho_{M(t)}\right] & =\frac{\mathrm{d}}{\mathrm{d} t} \int_{|x| \leq R(t)}\left(\frac{1}{2} \rho_{M(t)}(-\Delta)^{-s} \rho_{M(t)}+\frac{1}{2}|x|^{2} \rho_{M(t)}\right) \mathrm{d} x \\
& =\int_{|x| \leq R(t)}\left((-\Delta)^{-s} \rho_{M(t)}+\frac{|x|^{2}}{2}\right) \frac{\partial}{\partial t} \rho_{M(t)} \mathrm{d} x+R^{\prime}(t) \int_{|x|=R(t)} \frac{\rho_{M(t)}}{2}\left((-\Delta)^{-s} \rho_{M(t)}+|x|^{2}\right) \mathrm{d} x \\
& =\int_{|x| \leq R(t)}\left((-\Delta)^{-s} \rho_{M(t)}+\frac{|x|^{2}}{2}\right) \frac{\partial}{\partial t} \rho_{M(t)} \mathrm{d} x
\end{aligned}
$$

where the fact that $\rho_{M(t)}$ vanishes on the boundary $|x|=R(t)$ is used in the last step. From the characterization 20 of the local profile $\rho_{M(t)}$,

$$
\frac{\mathrm{d}}{\mathrm{d} t} H\left[\rho_{M(t)}\right]=\frac{N R(t)^{2}}{2(N-2 s)} \int \frac{\partial}{\partial t} \rho_{M(t)} \mathrm{d} x=\frac{N R(t)^{2}}{2(N-2 s)} M^{\prime}(t)=-\frac{N R(t)^{2}}{2(N-2 s)} P(t)^{-\delta} \int \rho^{r} \mathrm{~d} x .
$$

Using the characterization (20) and (21) again for the behaviour of $(-\Delta)^{-s} \rho_{M(t)}+|x|^{2} / 2$ inside and outside the ball $|x| \leq R(t)$, we finally get

$$
\frac{\mathrm{d}}{\mathrm{d} t} H\left[\rho_{M(t)}\right] \geq-P(t)^{-\delta} \int\left((-\Delta)^{-s} \rho_{M(t)}+\frac{|x|^{2}}{2}\right) \rho^{r} \mathrm{~d} x .
$$

With these preliminary formal computations, now we can show the exponential convergence of the relative entropy $H\left[\rho(t) \mid \rho_{M(t)}\right]$ as summarized in the following theorem. The result above holds only for $N=1$ since the essential entropy-entropy dissipation inequality (23) has only been proved in one dimension. As a result, the diffusion dominated regime (30) becomes $r>4-2 s$ or equivalently $\delta=(r-1) / \lambda-1>0$.

Theorem 3.3. Let $N=1$ and $\rho$ be a smooth solution of the one-dimensional fractional porous medium equation with absorption (31) with non-negative initial data $u_{0}$ and $s<1 / 2$. Let $\rho_{M(t)}$ be the Barenblatt profile with the same mass $M(t)$ as $\rho(t)$. Then we have that the relative entropy $H\left[\rho \mid \rho_{M(t)}\right]:=H[\rho]-$ $H\left[\rho_{M(t)}\right]$ decays to zero exponentially fast. That is, there is a constant $C$ depending on the mass $M_{0}:=$ $\left\|u_{0}\right\|_{1}$, the sup norm $\left\|u_{0}\right\|_{\infty}$, the exponents $r$, s and on $H\left[u_{0} \mid \rho_{M_{0}}\right]$, such that

$$
H\left[\rho(t) \mid \rho_{M(t)}\right] \leq C(1+t)^{2} \exp (-2 \min (1, \lambda \delta) t)
$$


Proof. The proof is based on a self-contained differential inequality of the relative entropy $H\left[\rho(t) \mid \rho_{M(t)}\right]$. From the two expressions (38) and (39) for the time derivative of the entropies, we have

$$
\begin{aligned}
\frac{\mathrm{d}}{\mathrm{d} t} H\left[\rho(t) \mid \rho_{M(t)}\right] & \leq-I[\rho(t)]-P(t)^{-\delta} \int \rho(t)^{r}(-\Delta)^{-s}\left(\rho(t)-\rho_{M(t)}\right) \mathrm{d} x \\
& \leq-I[\rho(t)]+P(t)^{-\delta}\left\|(-\Delta)^{-s / 2} \rho(t)^{r}\right\|_{2}\left\|(-\Delta)^{-s / 2}\left(\rho(t)-\rho_{M(t)}\right)\right\|_{2} .
\end{aligned}
$$

Using the one dimensional entropy-entropy dissipation inequality (23) and the relation (25), the differential inequality (41) becomes

$$
\frac{\mathrm{d}}{\mathrm{d} t} H\left[\rho(t) \mid \rho_{M(t)}\right] \leq-2 H\left[\rho(t) \mid \rho_{M(t)}\right]+\sqrt{2} P(t)^{-\delta}\left\|(-\Delta)^{-s / 2} \rho(t)^{r}\right\|_{2}\left(H\left[\rho(t) \mid \rho_{M(t)}\right]\right)^{1 / 2} .
$$

Applying the HLS inequality (7), i.e.

$$
\left\|(-\Delta)^{-s / 2} \rho(t)^{r}\right\|_{2} \leq C(s)\left\|\rho(t)^{r}\right\|_{\frac{2}{1+2 s}}=C(s)\|\rho(t)\|_{\frac{2 r}{1+2 s}}^{r} \leq C\left(\left\|u_{0}\right\|_{1},\left\|u_{0}\right\|_{\infty}, r, s\right),
$$

we get (recall that $P(t)=e^{\lambda t}$ )

$$
\frac{\mathrm{d}}{\mathrm{d} t} H\left[\rho(t) \mid \rho_{M(t)}\right] \leq-2 H\left[\rho(t) \mid \rho_{M(t)}\right]+C\left(\left\|u_{0}\right\|_{1},\left\|u_{0}\right\|_{\infty}, r, s\right) e^{-\lambda \delta t}\left(H\left[\rho(t) \mid \rho_{M(t)}\right]\right)^{1 / 2} .
$$

If the relative entropy $H\left[\rho(t) \mid \rho_{M(t)}\right]$ is strictly positive on any time interval $\left(t_{1}, t_{2}\right)$, then

$$
\frac{\mathrm{d}}{\mathrm{d} t}\left(H\left[\rho(t) \mid \rho_{M(t)}\right]\right)^{1 / 2} \leq-\left(H\left[\rho(t) \mid \rho_{M(t)}\right]\right)^{1 / 2}+C\left(\left\|u_{0}\right\|_{1},\left\|u_{0}\right\|_{\infty}, r, s\right) \exp (-\lambda \delta t), \quad t \in\left(t_{1}, t_{2}\right),
$$

By Gronwall's inequality, for any $t \in\left(t_{1}, t_{2}\right)$,

$$
H\left[\rho(t) \mid \rho_{M(t)}\right] \leq\left[e^{-\left(t-t_{1}\right)}\left(H\left[\rho\left(t_{1}\right) \mid \rho_{M\left(t_{1}\right)}\right]\right)^{1 / 2}+C\left(\left\|u_{0}\right\|_{1},\left\|u_{0}\right\|_{\infty}, r, s\right) e^{-\left(t-t_{1}\right)} \int_{t_{1}}^{t} \exp (\tau-\lambda \delta \tau) d \tau\right]^{2} .
$$

By choosing $t_{1}$ as small as possible, we have either $t_{1}=0$ or $H\left[\rho\left(t_{1}\right) \mid \rho_{M\left(t_{1}\right)}\right]=0$, and hence the following exponential convergence for any time $t>0$

$$
H\left[\rho(t) \mid \rho_{M(t)}\right] \leq C(1+t)^{2} \exp (-2 \min (1, \lambda \delta) t),
$$

where $C=C\left(H\left[u_{0} \mid \rho_{M_{0}}\right],\left\|u_{0}\right\|_{1},\left\|u_{0}\right\|_{\infty}, r, s\right)$.

Once the exponential convergence of the relative entropy is proved, the convergence in other metrics can be readily available, for instance the Wasserstein metric that is defined as

$$
W_{2}\left(\rho_{1}, \rho_{2}\right)=\left(\inf _{\pi \in \prod_{M}\left(\rho_{1}, \rho_{2}\right)} \iint_{\mathbb{R}^{N} \times \mathbb{R}^{N}}|x-y|^{2} \mathrm{~d} \pi(x, y)\right)^{1 / 2}
$$

with $\prod_{M}\left(\rho_{1}, \rho_{2}\right)$ being the set of all nonnegative Radon measures with total mass $M_{0}$ on $\mathbb{R}^{N} \times \mathbb{R}^{N}$ and marginals $\rho_{1}$ and $\rho_{2}$. Because of the displacement convexity of the entropy $H$, the following Talagrand inequality or transportation cost inequality

$$
W_{2}\left(\rho, \rho_{M}\right) \leq \sqrt{2\left(H[\rho]-H\left[\rho_{M}\right]\right)}
$$

holds true (see [11, Eq. (2.7)]), from which the Wasserstein distance between $\rho(t)$ and $\rho_{M(t)}$ also decreases exponentially fast as in the following corollary.

Corollary 3.4 (Convergence in Wasserstein metric). Under the same condition as in Theorem 3.3 .

$$
W_{2}\left(\rho(t), \rho_{M(t)}\right) \leq C(1+t) \exp (-\min (1, \lambda \delta) t)
$$

where $C$ has the same dependence as in Theorem 3.3. 


\subsection{Exponential convergence for general weak solutions}

In the previous subsection, the solutions are assumed to be smooth to justify all formal computations. However, the weak solutions obtained from the limiting sequences may not possess the required regularity and the exponential convergence of the relative entropy has to proved for the solutions of the regularized equation first, followed by a similar limiting procedure. To start, we consider the following regularized problem of Eq. (31):

$$
\frac{\partial \rho^{\epsilon}}{\partial t}=\nabla \cdot\left[\rho^{\epsilon} \nabla\left((-\Delta)^{-s} \rho^{\epsilon}+\frac{1}{2}|x|^{2}+\epsilon \log \rho^{\epsilon}\right)\right]-P(t)^{-\delta}\left(\rho^{\epsilon}\right)^{r}, \quad \rho^{\epsilon}(x, 0)=u_{0}(x) .
$$

It is easy to see that the solutions $\rho_{\epsilon}$ enjoy the same uniform $L^{p}$ bounds described in Proposition 3.1 . Because of the presence of linear diffusion, an associated regularized entropy is defined as

$$
H_{\epsilon}[\rho]=\int\left(\frac{1}{2} \rho(-\Delta)^{-s} \rho+\frac{|x|^{2}}{2} \rho+\epsilon \rho \log \rho\right) \mathrm{d} x
$$

together with the entropy dissipation rate

$$
I_{\epsilon}[\rho]=\int \rho\left|\nabla(-\Delta)^{-s} \rho+x+\epsilon \nabla \log \rho\right|^{2} \mathrm{~d} x .
$$

To make sure that the entropy $H_{\epsilon}\left[\rho^{\epsilon}(t)\right]$ is finite for the solution of Eq. (44), we first assume that the initial data has finite second moment, since this property propagates through any time, as we prove in the following lemma.

Lemma 3.5. If $u_{0}$ is a non-negative function in $L^{1}\left(\mathbb{R}^{N},\left(1+|x|^{2}\right) \mathrm{d} x\right) \cap L^{\infty}\left(\mathbb{R}^{N}\right)$, then a solution $\rho^{\epsilon}(t)$ of Eq. (44) stays in $L^{1}\left(\mathbb{R}^{N},\left(1+|x|^{2}\right) \mathrm{d} x\right)$ for all $t>0$ and

$$
\int|x|^{2} \rho^{\epsilon} \mathrm{d} x \leq C\left(\left\|u_{0}\right\|_{1},\left\|u_{0}\right\|_{\infty}, \int|x|^{2} u_{0} \mathrm{~d} x, N, s\right) .
$$

Proof. The computations below are based on the assumption that $\rho^{\epsilon}$ decays to zero fast enough in the far field, and can be made rigorous by using a cut-off function in space, see for instance [6, Lemma 2.1]. From the governing equation (44) for $\rho^{\epsilon}$,

$$
\begin{aligned}
\frac{\mathrm{d}}{\mathrm{d} t} \int \frac{|x|^{2}}{2} \rho^{\epsilon} \mathrm{d} x & =-\int \rho^{\epsilon}\left(x \cdot \nabla(-\Delta)^{-s} \rho^{\epsilon}+|x|^{2}\right) \mathrm{d} x-P(t)^{-\delta} \int \frac{|x|^{2}}{2}\left(\rho^{\epsilon}\right)^{r} \mathrm{~d} x+\epsilon \int \frac{|x|^{2}}{2} \Delta \rho^{\epsilon} \mathrm{d} x \\
& \leq-\int \rho^{\epsilon} x \cdot \nabla(-\Delta)^{-s} \rho^{\epsilon} \mathrm{d} x-\int|x|^{2} \rho^{\epsilon} \mathrm{d} x+\epsilon N M_{\epsilon}(t) .
\end{aligned}
$$

By the definition $(-\Delta)^{-s} \rho^{\epsilon}(x)=C_{N, s} \int|x-y|^{2 s-N} \rho^{\epsilon}(y) d y$ of the Riesz potential, the first term on the right hand of 46 becomes

$$
-\int \rho^{\epsilon} x \cdot \nabla(-\Delta)^{-s} \rho^{\epsilon} \mathrm{d} x=(N-2 s) C_{N, s} \iint \rho^{\epsilon}(x) \rho^{\epsilon}(y) x \cdot(x-y)|x-y|^{2 s-N-2} \mathrm{~d} y \mathrm{~d} x .
$$

The last double integral can be symmetrized by taking the average with the same expression when the variables $x$ and $y$ are exchanged. That is,

$$
-\int \rho^{\epsilon} x \cdot \nabla(-\Delta)^{-s} \rho^{\epsilon} \mathrm{d} x=\frac{N-2 s}{2} C_{N, s} \iint \rho^{\epsilon}(x) \rho^{\epsilon}(y)|x-y|^{2 s-N} \mathrm{~d} y \mathrm{~d} x=\frac{N-2 s}{2} \int \rho^{\epsilon}(-\Delta)^{-s} \rho^{\epsilon} \mathrm{d} x
$$

As a result, the rate of change of second moment can be bounded as

$$
\frac{\mathrm{d}}{\mathrm{d} t} \int \frac{|x|^{2}}{2} \rho^{\epsilon} \mathrm{d} x \leq(N-2 s) \int \rho^{\epsilon}(-\Delta)^{-s} \rho^{\epsilon} \mathrm{d} x-\int|x|^{2} \rho^{\epsilon} \mathrm{d} x+N M_{0} .
$$


By the Hardy-Littlewood-Sobolev inequality (7) and the uniform $L^{p}$ bounds described in Proposition 3.1 .

$$
\int \rho^{\epsilon}(-\Delta)^{-s} \rho^{\epsilon} \mathrm{d} x \leq\left\|(-\Delta)^{-\frac{s}{2}} \rho^{\epsilon}\right\|_{2}^{2} \leq C(N, s)\left\|\rho^{\epsilon}\right\|_{\frac{2 N}{N+2 s}}^{2} \leq C\left(\left\|u_{0}\right\|_{1},\left\|u_{0}\right\|_{\infty}, N, s\right) .
$$

This bound, combined with the differential inequality (47), leads to the desired estimate (45).

Lemma 3.5 assures that all three integrals in the entropy $H_{\epsilon}$ are finite along the flow of Eq. (44). Indeed, if the initial data $u_{0}$ is a nonnegative function in $L^{1}\left(\mathbb{R}^{N},\left(1+|x|^{2}\right) \mathrm{d} x\right) \cap L^{\infty}\left(\mathbb{R}^{N}\right)$, then both $\int|x|^{2} \rho^{\epsilon}(t) \mathrm{d} x$ and $\int \rho^{\epsilon}(-\Delta)^{-s} \rho^{\epsilon} \mathrm{d} x$ are finite. Since the Boltzmann entropy $\int \rho^{\epsilon}(t) \log \rho^{\epsilon}(t) \mathrm{d} x$ is bounded from above by $M_{\epsilon}(t) \log \left\|\rho_{M_{\epsilon}(t)}\right\|_{\infty}$, by Carleman type estimate (see [5, Lemma 2.2]), the uniform in time bound of the second order moments yields $\rho^{\epsilon}(t) \log \rho^{\epsilon}(t) \in L^{1}(\mathbb{R})$ at any time $t>0$. Moreover, Lemma 3.5 implies the mass confinement

$$
\lim _{R \rightarrow \infty} \sup _{\epsilon>0} \int_{|x|>R} \rho_{\epsilon}(t) \mathrm{d} x \leq \lim _{R \rightarrow \infty} \sup _{\epsilon>0} \frac{1}{R^{2}} \int_{|x|>R}|x|^{2} \rho_{\epsilon}(t) \mathrm{d} x=0 .
$$

This gives in particular the weak $L^{1}$ convergence of $\rho^{\epsilon}(t)$ to $\rho(t)$ thus in particular the convergence of $M_{\epsilon}(t)$ to $M(t)$. Using the lower semi-continuity of the entropy $H$ with respect to the weak*-convergence, we have

$$
H[\rho(t)] \leq \liminf _{\epsilon \rightarrow 0^{+}} H\left[\rho^{\epsilon}(t)\right]
$$

and

$$
\int|x|^{2} \rho(t) \mathrm{d} x \leq \liminf _{\epsilon \rightarrow 0^{+}} \int|x|^{2} \rho^{\epsilon}(t) \mathrm{d} x \leq C\left(N, s,\left\|u_{0}\right\|_{\infty},\left\|u_{0}\right\|_{1}, \int|x|^{2} u_{0} \mathrm{~d} x\right) .
$$

Now, arguing as in the proof of Lemma 3.5 we can show that higher order moments are also preserved, that will be needed in Section 3.4 to show the exponential convergence in $L^{1}$-norm.

Lemma 3.6 (Bounds on higher order moments). Let $n$ be a positive integer and $u_{0}$ be a non-negative function in $L^{1}\left(\mathbb{R}^{N},\left(1+|x|^{2 n}\right) \mathrm{d} x\right) \cap L^{\infty}\left(\mathbb{R}^{N}\right)$. Then a solution $\rho^{\epsilon}(t)$ to Eq. (44) with initial condition $u_{0}$ is also in $L^{1}\left(\mathbb{R}^{N},\left(1+|x|^{2 n}\right) \mathrm{d} x\right)$ for all $t>0$ and

$$
\int|x|^{2 n} \rho_{\epsilon} \mathrm{d} x \leq C\left(\left\|u_{0}\right\|_{1},\left\|u_{0}\right\|_{\infty}, \int|x|^{2 n} u_{0} \mathrm{~d} x, n, N, s\right) .
$$

Proof. From equation (31) satisfied by $\rho^{\epsilon}$,

$$
\frac{\mathrm{d}}{\mathrm{d} t} \int \frac{|x|^{2 n}}{2 n} \rho^{\epsilon} \mathrm{d} x \leq-\int|x|^{2 n-2} \rho^{\epsilon} x \cdot \nabla(-\Delta)^{-s} \rho^{\epsilon} \mathrm{d} x-\int|x|^{2 n} \rho^{\epsilon} \mathrm{d} x+\epsilon N(2 n-1) \int|x|^{2 n-2} \rho^{\epsilon} \mathrm{d} x .
$$

Using the fact that for any positive integer $n$ there exist a constant $K_{N, n}$ such that $\left(|x|^{2 n-2} x-|y|^{2 n-2} y\right)$. $(x-y) \leq K_{N, n}\left(|x|^{2 n-2}+|y|^{2 n-2}\right)|x-y|^{2}$, the non-local integral $-\int|x|^{2 n-2} \rho^{\epsilon} x \cdot \nabla(-\Delta)^{-s} \rho^{\epsilon}$ on the right hand side can be symmetrized as

$$
\begin{aligned}
& \frac{N-2 s}{2} C_{N, s} \iint \rho^{\epsilon}(x) \rho^{\epsilon}(y)\left(|x|^{2 n-2} x-|y|^{2 n-2} y\right) \cdot(x-y)|x-y|^{2 s-N-2} \mathrm{~d} y \mathrm{~d} x \\
\leq & \frac{(N-2 s) K_{N, n}}{2} \int|x|^{2 n-2} \rho^{\epsilon}(-\Delta)^{-s} \rho^{\epsilon} \mathrm{d} x .
\end{aligned}
$$

This integral can be further bounded as

$$
\begin{aligned}
\frac{(N-2 s) K_{N, n}}{2} \int|x|^{2 n-2} \rho^{\epsilon}(-\Delta)^{-s} \rho^{\epsilon} \mathrm{d} x & \leq \frac{1}{2} \int|x|^{2 n} \rho^{\epsilon} \mathrm{d} x+\tilde{K}(N, s, n) \int \rho^{\epsilon}\left|(-\Delta)^{-s} \rho^{\epsilon}\right|^{n} \mathrm{~d} x \\
& \leq \frac{1}{2} \int|x|^{2 n} \rho^{\epsilon} \mathrm{d} x+C\left(\left\|u_{0}\right\|_{1},\left\|u_{0}\right\|_{\infty}, n, N, s\right)
\end{aligned}
$$


where the $L^{\infty}$ bound of $\rho^{\epsilon}$ and the Hardy-Littlewood-Sobolev inequality is used in the last step. As a result, the rate of change of $2 n$-th moment becomes

$$
\frac{\mathrm{d}}{\mathrm{d} t} \int \frac{|x|^{2 n}}{2 n} \rho^{\epsilon} \mathrm{d} x \leq C\left(\left\|u_{0}\right\|_{1},\left\|u_{0}\right\|_{\infty}, n, N, s\right)-\frac{1}{2} \int|x|^{2 n} \rho_{\epsilon} \mathrm{d} x+\epsilon N(2 n-1) \int|x|^{2 n-2} \rho^{\epsilon} \mathrm{d} x .
$$

By induction on $n$, the differential inequality 49 implies that all moments of the solution $\rho^{\epsilon}$ up to order $2 n$ are bounded as well.

It is straightforward to show that $M_{\epsilon}(t) \leq M_{\epsilon}(0)=\left\|u_{0}\right\|_{1}=M_{0}$. We define the Barenblatt profile $\rho_{M_{\epsilon}(t)}^{\epsilon}$ as the minimizer of $H_{\epsilon}[\rho]$ over the set of admissible functions ${ }^{1}$

$$
\mathcal{Y}_{M_{\epsilon}(t)}=\left\{\eta \in L_{+}^{1}\left(\mathbb{R}^{N}\right):\|\eta\|_{1}=M_{\epsilon}(t), \int|x|^{2} \eta(x) \mathrm{d} x<\infty\right\} .
$$

This minimizer $\rho_{M_{\epsilon}(t)}^{\epsilon}$ exists and is unique from the convexity of the functional $H_{\epsilon}$ (see [15]). Moreover, $\rho_{M_{\epsilon}(t)}^{\epsilon}$ satisfies the Euler-Lagrange equation associated with $H_{\epsilon}$, that is

$$
(-\Delta)^{-s} \rho_{M_{\epsilon}(t)}^{\epsilon}+\frac{1}{2}|x|^{2}+\epsilon\left(\log \rho_{M_{\epsilon}(t)}^{\epsilon}+1\right)=\xi_{\epsilon}(t)
$$

for some constant $\xi_{\epsilon}(t)$. First, the counterpart of Lemma 2.9 relating $H^{-s}(\mathbb{R})$ norm the relative entropy still hold for the regularised entropy.

Lemma 3.7. Let $\eta$ be a non-negative function with total mass $M_{\epsilon}(t)$ such that $H_{\epsilon}[\eta]$ is finite, then $\left\|(-\Delta)^{-s / 2}\left(\eta-\rho_{M_{\epsilon}(t)}^{\epsilon}\right)\right\|_{2}^{2} \leq 2\left(H_{\epsilon}[\eta]-H_{\epsilon}\left[\rho_{M_{\epsilon}(t)}^{\epsilon}\right]\right)$.

Proof. Since $\rho_{M_{\epsilon}(t)}^{\epsilon}$ has the same mass $M_{\epsilon}(t)$ as $\eta$, from the characterization (50), we get

$$
0=\int \xi_{\epsilon}(t)\left(\eta-\rho_{M_{\epsilon}(t)}^{\epsilon}\right) \mathrm{d} x=\int\left(\eta-\rho_{M_{\epsilon}(t)}^{\epsilon}\right)\left((-\Delta)^{-s} \rho_{M_{\epsilon}(t)}^{\epsilon}+\frac{1}{2}|x|^{2}+\epsilon\left(\log \rho_{M_{\epsilon}(t)}^{\epsilon}+1\right)\right) \mathrm{d} x
$$

This identity can be rearranged to obtain the relation

$$
H_{\epsilon}[\eta]-H_{\epsilon}\left[\rho_{M_{\epsilon}(t)}^{\epsilon}\right]=\frac{1}{2}\left\|(-\Delta)^{-s / 2}\left(\eta-\rho_{M_{\epsilon}(t)}^{\epsilon}\right)\right\|_{2}^{2}+\epsilon \int \eta \log \frac{\eta}{\rho_{M_{\epsilon}(t)}^{\epsilon}} \mathrm{d} x .
$$

Applying Jensen's inequality (with then measure $\left.d \mu=\frac{\rho_{M_{\epsilon}(t)}^{\epsilon}}{M_{\epsilon}(t)} \mathrm{d} x\right)$,

$$
\int \eta \log \frac{\eta}{\rho_{M_{\epsilon}(t)}^{\epsilon}} \mathrm{d} x=M_{\epsilon}(t) \int \frac{\eta}{\rho_{M_{\epsilon}(t)}^{\epsilon}} \log \frac{\eta}{\rho_{M_{\epsilon}(t)}^{\epsilon}} d \mu(t) \geq M_{\epsilon}(t)\left(\int \frac{\eta}{\rho_{M_{\epsilon}(t)}^{\epsilon}} d \mu\right) \log \left(\int \frac{\eta}{\rho_{M_{\epsilon}(t)}^{\epsilon}} d \mu\right)=0 .
$$

Therefore, the desired inequality holds.

Although $\rho_{M_{\epsilon}(t)}^{\epsilon}$ is not expected to have explicit expressions as $\rho_{M}$ in (17), we can still get uniform bounds with respect to $\epsilon$ and $t>0$.

Proposition 3.8. The family of minimizers $\rho_{M_{\epsilon}(t)}^{\epsilon}$ is smooth and uniformly bounded in $L^{\infty}\left(\mathbb{R}^{N}\right)$ with respect to $\epsilon$.

\footnotetext{
${ }^{1}$ Here the superscript $\epsilon$ is used to distinguish it from $\rho_{M}$, the minimizer of $H[\rho]$ with total mass $M$.
} 
Proof. We first show that the minimizers are smooth for any $\epsilon$ small enough, and then show the bounds are uniform. Multiplying both sides of the Euler-Lagrange equation (50) with $\rho_{M_{\epsilon}(t)}$ and integrating on the whole space, we get

$$
\left(\xi_{\epsilon}(t)-\epsilon\right) M_{\epsilon}(t)=\frac{1}{2} \int \rho_{M_{\epsilon}(t)}^{\epsilon}(-\Delta)^{-s} \rho_{M_{\epsilon}(t)}^{\epsilon} \mathrm{d} x+H_{\epsilon}\left[\rho_{M_{\epsilon}(t)}^{\epsilon}\right] \leq H_{\epsilon}\left[\rho_{M_{\epsilon}(t)}^{\epsilon}\right] \leq H_{\epsilon}\left[\rho_{M_{\epsilon}(t)}\right],
$$

where the fact that $\rho_{M_{\epsilon}(t)}^{\epsilon}$ is the minimizer of $H_{\epsilon}$ is used in the last inequality. Since $M_{\epsilon}(t)$ converges to $M(t)>0, H_{\epsilon}\left[\rho_{M_{\epsilon}(t)}\right] / M_{\epsilon}(t)$ is uniformly bounded (for $\epsilon$ small), and so is $\xi_{\epsilon}-\epsilon$. Next again from the Euler-Langrange equation (50),

$$
\rho_{M_{\epsilon}(t)}^{\epsilon}=\exp \left(\frac{1}{\epsilon}\left(\xi_{\epsilon}(t)-(-\Delta)^{-s} \rho_{M_{\epsilon}(t)}^{\epsilon}-\frac{|x|^{2}}{2}\right)-1\right) \leq \exp \left(\frac{\xi_{\epsilon}(t)}{\epsilon}\right)
$$

which implies that $\rho_{M_{\epsilon}(t)}^{\epsilon} \in L^{\infty}(\mathbb{R})$ for any finite $\epsilon>0$.

To prove that $\rho_{M_{\epsilon}(t)}^{\epsilon}$ is smooth, we follow the main ideas from [14, Theorem 10] for a similar calculation for the case $s=1$. Indeed, using the interior regularity result for the fractional Laplacian stated in 34 , Theorem 1.1 and Corollary 3.5] (see also [8, Proposition 5.2]) we have $(-\Delta)^{-s} \rho_{M_{\epsilon}(t)}^{\epsilon} \in L^{\infty}(\mathbb{R})$ and

$$
\left\|(-\Delta)^{-s} \rho_{M_{\epsilon}(t)}^{\epsilon}\right\|_{C^{0,2 s}} \leq C\left(\left\|(-\Delta)^{-s} \rho_{M_{\epsilon}(t)}^{\epsilon}\right\|_{\infty}+\left\|\rho_{M_{\epsilon}(t)}^{\epsilon}\right\|_{\infty}\right)
$$

then from 51] we obtain $\rho_{M_{\epsilon}(t)}^{\epsilon} \in C^{0,2 s}$. Then we apply again the above mentioned regularity estimate of [34, saying that for $\alpha>0$ such that $\alpha+2 s$ not an integer,

$$
\left\|(-\Delta)^{-s} \rho_{M_{\epsilon}(t)}^{\epsilon}\right\|_{C^{0, \alpha+2 s}} \leq C\left(\left\|(-\Delta)^{-s} \rho_{M_{\epsilon}(t)}^{\epsilon}\right\|_{\infty}+\left\|\rho_{M_{\epsilon}(t)}^{\epsilon}\right\|_{C^{0, \alpha}}\right) .
$$

to find that $(-\Delta)^{-s} \rho_{M_{\epsilon}(t)}^{\epsilon} \in C^{\gamma}$ for any $\gamma<4 s$. Then by 51 we have $\rho_{M_{\epsilon}(t)}^{\epsilon} \in C^{\gamma}$ for any $\gamma<4 s$. Arguing iteratively, any order $\ell$ of differentiability for $(-\Delta)^{-s} \rho_{M_{\epsilon}(t)}^{\epsilon}$ (and then for $\rho_{M_{\epsilon}(t)}^{\epsilon}$ ) can be reached and hence $\rho_{M_{\epsilon}(t)}^{\epsilon} \in C^{\infty}$. we finally obtain $\rho_{M_{\epsilon}(t)}^{\epsilon}$ is actually $C^{\infty}$.

With the regularity properties we have just proved at hand, it is quite standard to obtain a uniform bound of $\rho_{M_{\epsilon}(t)}^{\epsilon}$ in time and $\epsilon>0$. Indeed, from equation 50 we have

$$
\begin{aligned}
0 & =\int \nabla\left(\rho_{M_{\epsilon}(t)}^{\epsilon}\right)^{p} \cdot \nabla\left[(-\Delta)^{-s} \rho_{M_{\epsilon}(t)}^{\epsilon}+\frac{1}{2}|x|^{2}+\epsilon\left(\log \rho_{M_{\epsilon}(t)}^{\epsilon}+1\right)\right] \mathrm{d} x \\
& \geq \int \nabla\left(\rho_{M_{\epsilon}(t)}^{\epsilon}\right)^{p} \cdot \nabla(-\Delta)^{-s} \rho_{M_{\epsilon}(t)}^{\epsilon} \mathrm{d} x-N \int\left(\rho_{M_{\epsilon}(t)}^{\epsilon}\right)^{p} \mathrm{~d} x \\
& \geq \frac{4 p}{(p+1)^{2}} \int\left|(-\Delta)^{\frac{1-s}{2}}\left(\rho_{M_{\epsilon}(t)}^{\epsilon}\right)^{\frac{p+1}{2}}\right|^{2} \mathrm{~d} x-N \int\left(\rho_{M_{\epsilon}(t)}^{\epsilon}\right)^{p} \mathrm{~d} x,
\end{aligned}
$$

where the Stroock-Varopoulos inequality (8) is used. Since a direct application of Nash-GagliardoNirenberg inequality 10 does not give bounds on $\left\|\rho_{M_{\epsilon}(t)}^{\epsilon}\right\|_{\infty}$, a classical Moser type iteration is used first for different norms. Then we apply the Nash-Gagliardo-Nirenberg inequality (10) with the choices

$$
\alpha=1-s, \quad l=\frac{2 p}{p+1}, \quad \theta=\frac{l}{2}=\frac{p}{p+1}, \quad m=\frac{N(2 p+1)}{(N-1+s)(p+1)}
$$

and $v=\left(\rho_{M_{\epsilon}(t)}^{\epsilon}\right)^{(p+1) / 2}$, in order to obtain

$$
\int\left(\rho_{M_{\epsilon}(t)}^{\epsilon}\right)^{p} \mathrm{~d} x \geq C(N, s, p)\|w\|_{m}^{\theta+1}=C(N, s, p)\left\|\rho_{M_{\epsilon}(t)}^{\epsilon}\right\|_{\mathrm{q}}^{(2 p+1) / 2}
$$


with $\mathbf{q}=\frac{N(2 p+1)}{2(N-1+s)}>p$. Then we finally have

$$
\left\|\rho_{M_{\epsilon}(t)}^{\epsilon}\right\|_{p}^{2 p} \geq \mathrm{C}\left\|\rho_{M_{\epsilon}(t)}^{\epsilon}\right\|_{\mathrm{q}}^{2 p+1}
$$

where the constant $\mathrm{C}=\mathrm{C}(N, s, p)$ verifies the asymptotic

$$
\mathrm{C}=\mathrm{C}(p, s) \sim \frac{C(s, N)}{p^{2}} \text { as } p \rightarrow \infty
$$

Now we can use 52 to iterate between different norms to get $\left\|\rho_{M_{\epsilon}(t)}^{\epsilon}\right\|_{\infty}$. We set $p_{0}=p$ and

$$
p_{k+1}=\sigma\left(p_{k}+\frac{1}{2}\right), \quad \sigma=\frac{N}{N-1+s} .
$$

We notice that, by our assumptions, the sequence $\left\{p_{k}\right\}$ is defined through

$$
p_{k}=A\left(\sigma^{k}-1\right)+p, \quad A=\frac{N}{2(1-s)}+p>0
$$

so that $p_{k}<p_{k+1}$ and $\lim _{k \rightarrow \infty} p_{k}=+\infty$. Then inequality 52 provides

$$
\mathrm{C}\left(N, s, p_{k}\right)\left\|\rho_{M_{\epsilon}(t)}^{\epsilon}\right\|_{p_{k+1}}^{2 p_{k}+1} \leq\left\|\rho_{M_{\epsilon}(t)}^{\epsilon}\right\|_{p_{k}}^{2 p_{k}}
$$

from which (recalling that $p_{k} \rightarrow \infty$ ),

$$
\left\|\rho_{M_{\epsilon}(t)}^{\epsilon}\right\|_{p_{k+1}} \leq \mathrm{C}\left(p_{k}, s\right)^{-\frac{1}{2 p_{k}+1}}\left\|\rho_{M_{\epsilon}(t)}^{\epsilon}\right\|_{p_{k} \frac{p_{k}}{p_{k+1}}}
$$

Since

$$
\lim _{k \rightarrow \infty} \mathrm{C}\left(p_{k}, s\right)^{-\frac{1}{2 p_{k}+1}}=1,
$$

setting $U_{k}=\left\|\rho_{M_{\epsilon}(t)}^{\epsilon}\right\|_{p_{k}}$, we can iterate as in [41, Proposition 5.4] and we can pass the limit and obtain the following bound of the $L^{\infty}$ norm of $\rho_{M_{\epsilon}(t)}^{\epsilon}$ in terms of its $L^{p}$ norm:

$$
\left\|\rho_{M_{\epsilon}(t)}^{\epsilon}\right\|_{\infty} \leq C\left\|\rho_{M_{\epsilon}(t)}^{\epsilon}\right\|_{p}^{\frac{2 p(1-s)}{1+2 p(1-s)}} .
$$

Since this inequality holds for all $p$, we can choose $p=1$, thus

$$
\left\|\rho_{M_{\epsilon}(t)}^{\epsilon}\right\|_{\infty} \leq C(N, s) M_{\epsilon}(t)^{\frac{2(1-s)}{1+2(1-s)}} \leq C\left(N, s, M_{0}\right)
$$

hence the proof follows.

We now focus on the convergence of the solution $\rho^{\epsilon}(t)$ of the regularised equation 44 towards the time-dependent profile $\rho_{M_{\epsilon}(t)}^{\epsilon}$, to recover the same rate as the regularization constant $\epsilon$ goes to zero. Arguing similarly to the smooth case in one dimension, we obtain

$$
\left.\frac{\mathrm{d}}{\mathrm{d} t} H_{\epsilon}\left[\rho^{\epsilon}\right]=-I_{\epsilon}\left[\rho^{\epsilon}\right]-P(t)^{-\delta} \int\left(\rho^{\epsilon}\right)^{r}\left[(-\Delta)^{-s} \rho^{\epsilon}+\frac{|x|^{2}}{2}+\epsilon\left(1+\log \rho^{\epsilon}\right)\right)\right] \mathrm{d} x,
$$

and

$$
\begin{aligned}
\frac{\mathrm{d}}{\mathrm{d} t} H_{\epsilon}\left[\rho_{M_{\epsilon}(t)}^{\epsilon}\right] & =\int\left((-\Delta)^{-s} \rho_{M_{\epsilon}(t)}^{\epsilon}+\frac{|x|^{2}}{2}+\epsilon\left(\log \rho_{M_{\epsilon}(t)}^{\epsilon}+1\right)\right) \frac{\partial \rho_{M_{\epsilon}(t)}^{\epsilon}}{\partial t} \mathrm{~d} x \\
& =\xi_{\epsilon}(t) \int \frac{\partial \rho_{M_{\epsilon}(t)}^{\epsilon}}{\partial t} \mathrm{~d} x=\xi_{\epsilon}(t) \frac{\mathrm{d}}{\mathrm{d} t} M_{\epsilon}(t),
\end{aligned}
$$


where the Euler-Lagrange equation (50) satisfied by the regularised profile $\rho_{M_{\epsilon}(t)}^{\epsilon}$ is used. From $\frac{\mathrm{d}}{\mathrm{d} t} M_{\epsilon}(t)=$ $-P(t)^{-\delta} \int\left(\rho^{\epsilon}\right)^{r} \mathrm{~d} x, \frac{d}{d t} H_{\epsilon}\left[\rho_{M_{\epsilon}(t)}^{\epsilon}\right]$ can be written as

$$
\begin{aligned}
\frac{d}{d t} H_{\epsilon}\left[\rho_{M_{\epsilon}(t)}^{\epsilon}\right] & =-\xi_{\epsilon}(t) P(t)^{-\delta} \int\left(\rho^{\epsilon}\right)^{r} \mathrm{~d} x \\
& =-P(t)^{-\delta} \int\left((-\Delta)^{-s} \rho_{M(t)}^{\epsilon}+\frac{1}{2}|x|^{2}+\epsilon\left(\log \rho_{M(t)}^{\epsilon}+1\right)\right)\left(\rho^{\epsilon}\right)^{r} \mathrm{~d} x .
\end{aligned}
$$

Therefore the time derivative of the relative entropy becomes

$$
\frac{d}{d t}\left(H_{\epsilon}\left[\rho^{\epsilon}\right]-H_{\epsilon}\left[\rho_{M_{\epsilon}(t)}^{\epsilon}\right]\right)=-I_{\epsilon}\left[\rho^{\epsilon}\right]-P(t)^{-\delta} \int\left(\rho^{\epsilon}\right)^{r}\left[(-\Delta)^{-s}\left(\rho^{\epsilon}-\rho_{M_{\epsilon}(t)}^{\epsilon}\right)+\epsilon \log \left(\rho^{\epsilon} / \rho_{M_{\epsilon}(t)}^{\epsilon}\right)\right] \mathrm{d} x .
$$

On the other hand, the logarithmic term in $(53)$ can be written as

$$
\int\left(\rho^{\epsilon}\right)^{r} \log \frac{\rho^{\epsilon}}{\rho_{M_{\epsilon}(t)}^{\epsilon}} \mathrm{d} x=\frac{\left\|\rho_{M_{\epsilon}(t)}^{\epsilon}\right\|_{r}^{r}}{r} \int\left(\frac{\rho^{\epsilon}}{\rho_{M_{\epsilon}(t)}^{\epsilon}}\right)^{r} \log \left(\frac{\rho^{\epsilon}}{\rho_{M_{\epsilon}(t)}^{\epsilon}}\right)^{r} d \mu, \quad d \mu=\frac{\left(\rho_{M(t)}^{\epsilon}\right)^{r}}{\left\|\rho_{M(t)}^{\epsilon}\right\|_{r}^{r}} \mathrm{~d} x .
$$

By Jensen's inequality again we have

$$
\begin{aligned}
\int\left(\rho^{\epsilon}\right)^{r} \log \frac{\rho^{\epsilon}}{\rho_{M_{\epsilon}(t)}^{\epsilon}} \mathrm{d} x & \geq \frac{\left\|\rho_{M_{\epsilon}(t)}^{\epsilon}\right\|_{r}^{r}}{r}\left[\int\left(\frac{\rho^{\epsilon}}{\rho_{M_{\epsilon}(t)}^{\epsilon}}\right)^{r} \mathrm{~d} \mu\right] \log \left[\int\left(\frac{\rho^{\epsilon}}{\rho_{M_{\epsilon}(t)}^{\epsilon}}\right)^{r} \mathrm{~d} \mu\right] \\
& \geq-\frac{\left\|\rho_{M_{\epsilon}(t)}^{\epsilon}\right\|_{r}^{r}}{r} \frac{1}{e}
\end{aligned}
$$

where the fact that $f(z)=z \log z \geq-1 / e$ for $z \geq 0$ is used in the last step. As a result, by Proposition 3.8 we find

$$
-\epsilon \int\left(\rho^{\epsilon}\right)^{r} \log \frac{\rho^{\epsilon}}{\rho_{M_{\epsilon}(t)}^{\epsilon}} \mathrm{d} x \leq \epsilon C\left\|\rho_{M_{\epsilon}(t)}^{\epsilon}\right\|_{r}^{r} \leq \epsilon C_{1}\left(\left\|u_{0}\right\|_{1}, r, s\right)
$$

Now we notice that (see [11, Proposition 2.4] for more details) the entropy-entropy dissipation inequality is still valid for $H_{\epsilon}$, i.e.,

$$
H_{\epsilon}\left[\rho^{\epsilon}\right]-H_{\epsilon}\left[\rho_{M_{\epsilon}(t)}^{\epsilon}\right] \leq \frac{1}{2} I_{\epsilon}\left[\rho^{\epsilon}\right] .
$$

Consequently we get the following self-contained differential inequality from (53),

$$
\begin{aligned}
& \frac{d}{d t}\left(H_{\epsilon}\left[\rho^{\epsilon}\right]-H_{\epsilon}\left[\rho_{M_{\epsilon}(t)}^{\epsilon}\right]\right) \leq-2\left(H_{\epsilon}\left[\rho^{\epsilon}\right]-H_{\epsilon}\left[\rho_{M(t)}^{\epsilon}\right]\right) \\
& \quad+C\left(\left\|u_{0}\right\|_{1},\left\|u_{0}\right\|_{\infty}, r, s\right) P(t)^{-\delta}\left(H_{\epsilon}\left[\rho^{\epsilon}\right]-H_{\epsilon}\left[\rho_{M(t)}^{\epsilon}\right)^{1 / 2}+\epsilon C_{1}\left(\left\|u_{0}\right\|_{1}, r, s\right) P(t)^{-\delta} .\right.
\end{aligned}
$$

Now we can prove Theorem 2.10 about the exponential convergence of relative entropy of general weak solutions, by taking the limit as $\epsilon$ goes to zero.

Proof of Theorem 2.10. If we set $f_{\epsilon}(t)=H_{\epsilon}\left[\rho^{\epsilon}(t)\right]-H_{\epsilon}\left[\rho_{M_{\epsilon}(t)}^{\epsilon}\right.$, then (54) implies that $f_{\epsilon}$ satisfies the differential inequality

$$
f_{\epsilon}^{\prime}(t) \leq-2 f+C_{1} e^{-\lambda \delta t} f^{1 / 2}+\epsilon C_{2} e^{-\lambda \delta t} .
$$

From Lemma 3.9 below, we have

$$
\liminf _{\epsilon \rightarrow 0^{+}}\left(H_{\epsilon}\left[\rho^{\epsilon}(t)\right]-H_{\epsilon}\left[\rho_{M_{\epsilon}(t)}^{\epsilon}\right]\right) \leq C(1+t)^{2} e^{-2 \min (1, \lambda \delta)}
$$

and our aim now is to show the above limit on the left hand side is larger than $H[\rho(t)]-H\left[\rho_{M(t)}\right]$. Since $\rho^{\epsilon}$ is uniformly bounded in $L^{p}(\mathbb{R})$ with finite second moment, $\left|\int \rho^{\epsilon} \log \rho^{\epsilon}\right|$ is also uniformly bounded. From 
the lower semi-continuity of the entropy $H[\rho]$ ([11, Theorem 3.1]), when $\epsilon$ goes to zero, $\rho^{\epsilon}(t)$ converges to $\rho(t)$ in weak $L^{1}$, and

$$
\liminf _{\epsilon \rightarrow 0^{+}} H_{\epsilon}\left[\rho^{\epsilon}(t)\right] \geq \liminf _{\epsilon \rightarrow 0^{+}} H\left[\rho^{\epsilon}(t)\right]=H[\rho(t)] .
$$

For the other term $H_{\epsilon}\left[\rho_{M_{\epsilon}(t)}\right]$, we can show that the limit as $\epsilon$ goes to zero actually exist. If we define

$$
\mathcal{H}[\eta]=\pi M_{\epsilon}(t)^{-2} \int|x|^{2} \eta \mathrm{d} x+\int \eta \log \eta \mathrm{d} x
$$

for any function $\eta$ in $L^{1}\left(\mathbb{R},\left(1+|x|^{2}\right) \mathrm{d} x\right)$ such that $\int \eta(x) \mathrm{d} x=M_{\epsilon}(t)$, then $\mathcal{H}[\eta]$ is non-negative and $\mathcal{H}[\eta]=0$ only when $\eta(x)=\exp \left(-\pi|x|^{2} / M_{\epsilon}(t)^{2}\right)$. If $\epsilon$ is restricted to be less than $\frac{1}{2 \pi} M_{\epsilon}(t)$,

$$
\begin{aligned}
H_{\epsilon}\left[\rho_{M_{\epsilon}(t)}^{\epsilon}\right] & =\epsilon \mathcal{H}\left[\rho_{M_{\epsilon}(t)}^{\epsilon}\right]+\left(1-2 \epsilon \pi M_{\epsilon}(t)^{-2}\right) H\left[\rho_{M_{\epsilon}(t)}^{\epsilon}\right]+\epsilon \pi M_{\epsilon}(t)^{-2} \int \rho_{M_{\epsilon}(t)}^{\epsilon}(-\Delta)^{-s} \rho_{M_{\epsilon}(t)}^{\epsilon} \mathrm{d} x \\
& \geq\left(1-2 \epsilon \pi M_{\epsilon}(t)^{-2}\right) H\left[\rho_{M_{\epsilon}(t)}\right]
\end{aligned}
$$

which implies that

$$
\liminf _{\epsilon \rightarrow 0} H_{\epsilon}\left[\rho_{M_{\epsilon}(t)}^{\epsilon}\right] \geq \lim _{\epsilon \rightarrow 0}\left(1-2 \epsilon \pi M_{\epsilon}(t)^{-2}\right) H\left[\rho_{M_{\epsilon}(t)}\right]=H\left[\rho_{M(t)}\right] .
$$

Here in the last step the limit exists because the entropies $H\left[\rho_{M_{\epsilon}(t)}\right], H\left[\rho_{M(t)}\right]$ depend only on the masses $M_{\epsilon}(t), M(t)$ and $M_{\epsilon}(t) \rightarrow M(t)$ (recall that we have the explicit form of the Barenblatt profile (17)). Moreover, due to the minimality of $\rho_{M_{\epsilon}(t)}$ and the fact that the Boltzmann entropy $\int \rho_{M_{\epsilon}(t)} \log \rho_{M_{\epsilon}(t)} \mathrm{d} x$ is uniformly bounded from above w.r. to $\epsilon$ (implying that $\rho_{M_{\epsilon}(t)} \log \rho_{M_{\epsilon}(t)}$ is uniformly bounded in $L^{1}(\mathbb{R})$ ), we have

$$
H_{\epsilon}\left[\rho_{M_{\epsilon}(t)}^{\epsilon}\right] \leq H_{\epsilon}\left[\rho_{M_{\epsilon}(t)}\right]=H\left[\rho_{M_{\epsilon}(t)}\right]+\epsilon \int \rho_{M_{\epsilon}(t)} \log \rho_{M_{\epsilon}(t)} \mathrm{d} x
$$

then

$$
\limsup _{\epsilon \rightarrow 0} H_{\epsilon}\left[\rho_{M_{\epsilon}(t)}^{\epsilon}\right] \leq H\left[\rho_{M(t)}\right]
$$

which finally gives

$$
\lim _{\epsilon \rightarrow 0} H_{\epsilon}\left[\rho_{M_{\epsilon}(t)}^{\epsilon}\right]=H\left[\rho_{M(t)}\right] .
$$

Putting all these together, we get the exponential convergence 440 , which is exactly (27) via the change of variables 15 . The convergence of $u(\tau)-u_{M(\tau)}$ in the $H^{-s}(\mathbb{R})$ norm is then a direct consequence of 25$)$.

In the previous theorem we used the following technical lemma.

Lemma 3.9. Let $f_{\epsilon}(t)$ be a family of non-negative functions defined on $t \in[0, \infty)$ satisfying the differential inequalities $f_{\epsilon}^{\prime}(t) \leq F\left(f_{\epsilon}(t), t, \epsilon\right)$. Here $\epsilon \in(0, \bar{\epsilon}]$ for some $\bar{\epsilon}>0$ and

$$
F(f, t, \epsilon)=-2 f+C_{1} e^{-\lambda \delta t} f^{\frac{1}{2}}+\epsilon C_{2} e^{-\lambda \delta t}
$$

for some positive constants $C_{1}, C_{2}$ and $\lambda \delta>0$. Then the following statements hold.

(a) The family of functions $f_{\epsilon}$ satisfies the bound

$$
f_{\epsilon}(t) \leq e^{-2 t} f_{\epsilon}(0)+C_{4}(1+t) e^{-\min (2, \lambda \delta) t}
$$

where

$$
C_{4}=C_{1} C_{3}^{1 / 2}+\bar{\epsilon} C_{2} \quad \text { and } \quad C_{3}=\max \left\{\sup _{\epsilon \in(0, \bar{\epsilon}]} f_{\epsilon}(0), C_{1}^{2}, \bar{\epsilon} C_{2}\right\}
$$


(b) we have

$$
\liminf _{\epsilon \rightarrow 0^{+}} f_{\epsilon}(t) \leq C(1+t)^{2} e^{-2 \min (1, \lambda \delta) t} .
$$

for some positive constant $C$ depending on $\bar{\epsilon}, C_{1}, C_{2}$ and $\lim _{\epsilon \rightarrow 0^{+}} f_{\epsilon}(0)$.

Proof. (a) First by the classical comparison principle of ODEs between $f_{\epsilon}(t)$ and the constant $C_{3}$ defined above, $F\left(C_{3}, t, \epsilon\right) \leq 0$ and hence $f_{\epsilon}(t)$ is uniformly bounded above by $C_{3}$. Therefore, $f_{\epsilon}(t)$ satisfies the simpler differential inequality

$$
f_{\epsilon}^{\prime} \leq-2 f_{\epsilon}+C_{4} e^{-\lambda \delta t}
$$

The desired inequality is obtained by integrating the equivalent inequality $\frac{\mathrm{d}}{\mathrm{d} t}\left(e^{2 t} f_{\epsilon}(t)\right) \leq C_{4} e^{(2-\lambda \delta) t}$.

(b) In addition to the comparison principle of differential inequalities, continuous dependence of solutions of ODEs on the parameter $\epsilon$ will also be used, where the main barrier of non-Lipschitz continuity of $F(f, t, \epsilon)$ at $f=0$ in applying these techniques is considered separately.

First define $g_{\epsilon}(t)$ to be the solution of the ODE $g_{\epsilon}^{\prime}(t)=F\left(g_{\epsilon}(t), t, \epsilon\right)$ with $g_{\epsilon}(0)=f_{\epsilon}(0)$. Then from $g_{\epsilon}^{\prime}(t) \geq-2 g_{\epsilon}(t)$, we have $g_{\epsilon}(t) \geq g_{\epsilon}(0) e^{-2 t}$ on any finite interval $[0, T]$ and $F\left(g_{\epsilon}, t, \epsilon\right)$ is now Lipschitz on $\left(g_{\epsilon}(0), \infty\right) \times(0, \infty) \times(0, \bar{\epsilon})$. Then by the continuous dependence of ODEs, the limit $g(t)=\lim _{\epsilon \rightarrow 0^{+}} g_{\epsilon}(t)$ exists, and $g(t)$ satisfies $g^{\prime}(t)=F(g(t), t, 0)$ with the initial condition $g(0)=\liminf _{\epsilon \rightarrow 0^{+}} f_{\epsilon}(0)$. Moreover, $g(t) \leq C(1+t)^{2} e^{-2 \min (1, \lambda \delta) t}$ by the same argument as in the proof of Theorem 3.3 .

Next we apply the comparison principle between $f_{\epsilon}$ and $g_{\epsilon}$. If $f_{\epsilon}(t)<f_{\epsilon}(0) e^{-2 t}$, we get $f_{\epsilon}(t) \leq g_{\epsilon}(t)$; otherwise if $f_{\epsilon}(t) \geq f_{\epsilon}(0) e^{-2 t}$ on some interval $\left[t_{0}, t_{1}\right]$, the comparison principle between $f_{\epsilon}(t)$ and $g_{\epsilon}(t)$ still applies, where the initial condition $f_{\epsilon}(t) \leq g_{\epsilon}(t)$ can be enforced at $t=t_{0}$ by choosing $t_{0}$ as small as possible. In either case, the relation $f_{\epsilon}(t) \leq g_{\epsilon}(t)$ is satisfied on any finite interval $[0, T]$, on which

$$
\liminf _{\epsilon \rightarrow 0^{+}} f_{\epsilon}(t) \leq \lim _{\epsilon \rightarrow 0^{+}} g_{\epsilon}(t)=g(t) .
$$

\subsection{Comments on $L^{2}$ and $L^{1}$ decays}

Once the exponential convergence in the relative entropy $H\left[\rho(t) \mid \rho_{M(t)}\right]$ (or $H\left[u(\tau) \mid u_{M(\tau)}\right]$ ) is established, the convergence in $\dot{H}^{-s}(\mathbb{R})$ norm or in the Wasserstein metric $W_{2}$ is straightforward, using the inequalities (25) and (43). However, the convergence in other common norms like $L^{1}(\mathbb{R})$ or $L^{2}(\mathbb{R})$ is less obvious. In the case of classical heat equation, the convergence in $L^{1}(\mathbb{R})$ norm can be derived from the relative Boltzmann entropy, using the well-known Csiszár-Kullback inequality. For the entropy $H[\rho]$ used here, the convergence in $L^{1}(\mathbb{R})$ or $L^{2}(\mathbb{R})$ norm can not be established directly using similar inequalities, and has to rely on interpolation lemma like below [11, Theorem 3.4], with additional assumptions on the Hölder regularity of the solution $\rho(t)$.

Lemma 3.10 (Interpolation between norms). Let $0<\mathrm{a} \leq 1,0<s<N / 2$ and $0<\mathrm{a}<\alpha / 2$. There exists a constant $C$ depending on $N, s$ and a only, such that

$$
\|u\|_{2} \leq C\left\|(-\Delta)^{-s / 2} u\right\|_{2}^{\sigma_{1}}[u]_{\alpha}^{\sigma_{2}}\|u\|_{1}^{\sigma_{3}}
$$

for any function $u \in L^{1}\left(\mathbb{R}^{N}\right) \cap C^{\alpha}\left(\mathbb{R}^{N}\right)$ with

$$
\sigma_{1}=\frac{\mathrm{a}}{\mathrm{a}+s}, \quad \sigma_{2}=\frac{s(N+2 \mathrm{a})}{2(N+\alpha)(s+\mathrm{a})}, \quad \sigma_{3}=\frac{s(N+2 \alpha-2 \mathrm{a})}{2(N+\alpha)(s+\mathrm{a})},
$$

where $[\cdot]_{\alpha}$ denotes the Hölder seminorm. 
The explicit expression (17) of $\rho_{M(t)}$ implies that $\rho_{M(t)}$ is $(1-s)$-Hölder continuous with $\left[\rho_{M(t)}\right]_{\alpha}$ depending only on $M(t)$. If the solution $\rho(t)$ is also $\alpha$-Hölder continuous with uniform in time bound of $[\rho(t)]_{\alpha}$, then by choosing $u=\rho(t)-\rho_{M(t)}$ in Lemma 3.10 (with $N=1$ ) and $0<\mathrm{a}<\min (\alpha, 1-s) / 2$, we get

$$
\left\|\rho(t)-\rho_{M(t)}\right\|_{2} \leq C\left\|(-\Delta)^{-s / 2}\left(\rho(t)-\rho_{M(t)}\right)\right\|_{2}^{\sigma_{1}}\left[\rho(t)-\rho_{M(t)}\right]_{\alpha}^{\sigma_{2}}\left\|\rho(t)-\rho_{M(t)}\right\|_{1}^{\sigma_{3}} .
$$

Using (25), the uniform in time bound of $\left[\rho(t)-\rho_{M(t)}\right]_{\alpha}$ and the fact that $\|\rho(t)\|_{L^{1}}+\left\|\rho_{M(t)}\right\|_{1} \leq 2 M_{0}$, we get

$$
\left\|\rho(t)-\rho_{M(t)}\right\|_{2} \leq C\left(H\left[\rho \mid \rho_{M(t)}\right]\right)^{\frac{\sigma_{1}}{2}},
$$

for some constant $C$ depending on $\alpha$, a, $M_{0}$. Combining this last inequality with the exponential convergence (40) of the relative entropy $H\left[\rho(t) \mid \rho_{M(t)}\right]$ we obtain the following convergence rate in $L^{2}$ norm,

$$
\left\|\rho(t)-\rho_{M(t)}\right\|_{2} \leq C(1+t)^{\sigma_{1}} \exp \left(-\sigma_{1} \min (1, \lambda \delta) t\right) .
$$

The convergence in the $L^{1}(\mathbb{R})$ norm is more involved, because uniform bounds in higher order moments are needed. Arguing as in [11, Corollary 3.5] and using [13, Lemma 2.24], we find the following interpolation between the $L^{1}\left(\mathbb{R}^{N}\right)$ and $L^{2}\left(\mathbb{R}^{N}\right)$ norms,

$$
\left\|\rho(t)-\rho_{M(t)}\right\|_{1} \leq C(N, n)\left\|\rho(t)-\rho_{M(t)}\right\|_{2}^{\frac{4 n}{4 n+1}}\left(\int|x|^{2 n}\left(\rho+\rho_{M(t)}\right) \mathrm{d} x\right)^{\frac{1}{1+4 n}} .
$$

Provided that $u_{0} \in L^{1}\left(\mathbb{R},\left(1+|x|^{2 n}\right) \mathrm{d} x\right) \cap L^{\infty}(\mathbb{R})$, Lemma 3.6 guarantees uniform in time bound of the $2 n$-th moment. Therefore, the convergence in $L^{2}(\mathbb{R})$ norm in 55 yields finally to the convergence in $L^{1}(\mathbb{R})$, i.e.

$$
\left\|\rho(t)-\rho_{M(t)}\right\|_{1} \leq C(1+t)^{\frac{4 n \sigma_{1}}{1+4 n}} \exp \left(-\frac{4 n \sigma_{1}}{1+4 n} \min (1, \lambda \delta) t\right) .
$$

Using the similarity variables, the $L^{1}$ convergence for the solutions of Eq. (3) can also be derived,

$$
\left\|u(\tau)-U_{M(\tau)}\right\|_{1} \leq C(1+\log \tau)^{\frac{4 n \sigma_{1}}{1+4 n}}(1+\tau)^{-\frac{4 n \sigma_{1}}{1+4 n} \min \left(\frac{1}{\lambda}, \delta\right)} .
$$

This convergence, together with the decay of $L^{\infty}(\mathbb{R})$ norm of the solution $u(\tau)$ in Proposition 2.4, implies the convergence in $L^{p}(\mathbb{R})$ for any $p \in(1, \infty)$, although the rate is unlikely to be optimal.

\section{Fractional diffusion equation with convection}

In this section we focus on the long term asymptotic behaviours of solutions to the nonlocal porous medium equation (4) with convection. As in the previous case with absorption, basic properties of the solutions will be reviewed first, followed by the proof of exponential convergence of relative entropy for smooth solutions and then for more general weak solutions through limiting process. Detailed proofs in some of the statements below will be omitted, if they are straightforward or similar to the case with absorption.

Using the same similarity variables (15), Eq. (4) becomes

$$
\rho_{t}-\nabla \cdot\left(\rho \nabla(-\Delta)^{-s} \rho+x \rho\right)=-P(t)^{-\theta} \mathbf{b} \cdot \nabla \rho^{q},
$$

with $P(t)=1+\lambda \tau=e^{\lambda t}, \theta=(N(q-1)+1) / \lambda-1$ and $\rho(x, 0)=u_{0}(x)$. Here we are interested in the diffusion dominated regime with $\theta>0$, or equivalently $q>(2 N+1-2 s) / N$. The divergence structure of Eq. (56) implies the conservation of total mass, namely for all $t \geq 0$,

$$
M_{0}:=\int u_{0}(x) \mathrm{d} x=\int \rho(x, t) \mathrm{d} x .
$$

Arguing in the same way as in Proposition 3.1. we get the same estimates. 
Proposition 4.1. Let $\rho$ be a solution to problem (56). Then estimates (32), (33), (34), (35) still hold.

We will focus on the one dimensional case below with $\mathbf{b}=1$. It turns out that in this special case, weak solutions to Eq. (4) (and hence weak solutions to Eq. (56)) are unique, by introducing the variable in the integrated form, i.e.,

$$
v(x, \tau)=\int_{-\infty}^{x} u(y, \tau) \mathrm{d} y \text { for } \tau \geq 0, x \in \mathbb{R} .
$$

As a result, if $u$ satisfies Eq. (4), then $v$ satisfies the equation

$$
v_{\tau}+\left|v_{x}\right|(-\Delta)^{1-s} v+\left|v_{x}\right|^{q}=0
$$

with the initial data $v(x, 0)=v_{0}(x)=\int_{-\infty}^{x} u_{0}(y) \mathrm{d} y$. The mass conservation of $u$ implies the following boundary conditions for $v$,

$$
\lim _{x \rightarrow-\infty} v(x, \tau)=0, \quad \lim _{x \rightarrow+\infty} v(x, \tau)=M_{0}
$$

for all $\tau \geq 0$. In fact, $v$ is a viscosity solution of Eq. (57) by following the procedures in [38, Proposition 4.2] (see also [37, Section 8]). The uniqueness of these viscosity solutions is then guaranteed by the comparison principle proved in [16, Theorem 6.1], yielding to the following result.

Lemma 4.2. Let $u_{0}$ be a nonnegative function in $L^{1}(\mathbb{R})$. Then there exist a unique weak solution to Eq. (4) with initial data $u_{0}$.

We now prove the exponential convergence of the relative entropy between the solution $\rho$ and its Barenblatt profile $\rho_{M_{0}}$. Formally, if $\rho$ is a smooth solution to Eq. (56), then

$$
\frac{\mathrm{d}}{\mathrm{d} t} H[\rho]=-I[\rho]-P(t)^{-\theta} \int\left(\rho^{q}\right)_{x}\left((-\Delta)^{-s} \rho+\frac{1}{2}|x|^{2}\right) \mathrm{d} x .
$$

Now the key step to obtain a self-contained differential inequality is to relate the last integral above to $I[\rho]$ (instead of $H[\rho]$ as in the absorption case) via integration by parts and the Cauchy-Schwarz inequality. That is,

$$
\begin{aligned}
-P(t)^{-\theta} \int\left(\rho^{q}\right)_{x}\left[\frac{x^{2}}{2}+(-\Delta)^{-s} \rho\right] \mathrm{d} x & =P(t)^{-\theta} \int \rho^{q}\left[\frac{x^{2}}{2}+(-\Delta)^{-s} \rho\right]_{x} \mathrm{~d} x \\
& \leq P(t)^{-\theta}\left(\int_{\mathbb{R}}|\rho|^{2 q-1} \mathrm{~d} x\right)^{1 / 2} I[\rho]^{1 / 2} \\
& \leq C\left(\left\|u_{0}\right\|_{1},\left\|u_{0}\right\|_{\infty}, q, s\right) P(t)^{-\theta} I[\rho]^{1 / 2} .
\end{aligned}
$$

Since the total mass is conserved, $H\left[\rho_{M_{0}}\right]$ is a constant and the above computation implies

$$
\frac{\mathrm{d}}{\mathrm{d} t} H\left[\rho(t) \mid \rho_{M_{0}}\right] \leq-I[\rho(t)]+C P(t)^{-\theta} I[\rho(t)]^{\frac{1}{2}},
$$

from which the exponential convergence of the relative entropy can be proved as in the following theorem.

Theorem 4.3. Let $N=1, q>3-2 s$ and $\rho$ be the unique weak solution of the one-dimensional nonlocal porous medium equation (56) with non-negative initial data $u_{0} \in L^{1}\left(\mathbb{R},\left(1+|x|^{2}\right) d x\right) \cap L^{\infty}(\mathbb{R})$ and $s<1 / 2$. Assume that $\rho$ is smooth and let $\rho_{M_{0}}$ be the Barenblatt profile with total conserved mass $M_{0}$. Then the relative entropy $H\left[\rho \mid \rho_{M_{0}}\right]$ decays to zero exponentially fast. More precisely, there is a constant $C$ depending on $\left\|u_{0}\right\|_{1},\left\|u_{0}\right\|_{\infty}, H\left[u_{0} \mid \rho_{M_{0}}\right]$, q and $s$, such that

$$
H\left[\rho(t) \mid \rho_{M_{0}}\right] \leq C(1+t)^{2} \exp (-2 \min (1, \lambda \theta) t) .
$$


Proof. Define the function $f(\sigma)=-\sigma+C P(t)^{-\theta} \sigma^{1 / 2}$ for non-negative $\sigma$. Then $f$ is decreasing on the interval $\left[C^{2} P(t)^{-2 \theta} / 4, \infty\right)$. If $H\left[\rho(t) \mid \rho_{M_{0}}\right] \leq C^{2} P(t)^{-2 \theta} / 8$, then the bound $(59)$ holds true. Otherwise if $H\left[\rho(t) \mid \rho_{M_{0}}\right]>C^{2} P(t)^{-2 \theta} / 8$ at any time $t$, by the entropy-entropy dissipation inequality (23),

$$
C^{2} P(t)^{-2 \theta} / 4<2 H\left[\rho(t) \mid \rho_{M_{0}}\right] \leq I[\rho(t)]
$$

and hence $f(I[\rho(t)]) \leq f\left(2 H\left[\rho(t) \mid \rho_{M_{0}}\right)\right.$ by the monotonicity of $f$. Therefore Eq. (58) implies

$$
\frac{\mathrm{d}}{\mathrm{d} t} H\left[\rho \mid \rho_{M_{0}}\right] \leq f\left(2 H\left[\rho \mid \rho_{M_{0}}\right]\right)=-2 H\left[\rho \mid \rho_{M_{0}}\right]+\sqrt{2} C P(t)^{-\theta}\left(H\left[\rho \mid \rho_{M_{0}}\right]\right)^{1 / 2} .
$$

This differential inequality can be solved in the same way as in Theorem 3.3 to obtain the exponential bound (59) of the relative entropy.

To show the exponential convergence of general weak solutions of Eq. (56), similarly we consider the one-dimensional regularised problem

$$
\frac{\partial \rho^{\epsilon}}{\partial t}=\nabla \cdot\left[\rho^{\epsilon} \nabla\left((-\Delta)^{-s} \rho^{\epsilon}+\frac{1}{2}|x|^{2}+\epsilon \log \rho^{\epsilon}\right)\right]-P(t)^{-\theta} \frac{\partial}{\partial x}\left(\rho^{\epsilon}\right)^{q}, \quad \rho^{\epsilon}(x, 0)=u_{0}(x) .
$$

Many properties of the solution $\rho^{\epsilon}$ can be established in a similar way in the absorption case, such as the counterpart of Lemma 3.5, the confinement of total mass and the weak $L^{1}$ convergence of $\rho^{\epsilon}(t)$ to the weak solution $\rho(t)$ as $\epsilon$ goes to zero. Moreover, from the governing equation $(60)$ satisfied by $\rho^{\epsilon}$, we get

$$
\frac{\mathrm{d}}{\mathrm{d} t} H_{\epsilon}\left[\rho^{\epsilon}(t) \mid \rho_{M_{0}}^{\epsilon}\right] \leq-I_{\epsilon}\left[\rho^{\epsilon}(t)\right]+C P(t)^{-\theta} I_{\epsilon}\left[\rho^{\epsilon}(t)\right]^{1 / 2} .
$$

Using the same technique as in Theorem 4.3, we obtain the exponential convergence of $H_{\epsilon}\left[\rho^{\epsilon}(t) \mid \rho_{M_{0}}^{\epsilon}\right]$, and hence the same bound (59) for general weak solutions by taking the limit as $\epsilon$ goes to zero, providing the proof of Theorem 2.11 for the convergence of relative entropy for the original equation (4). Finally, by assuming uniform in time Hölder seminorm of the solution $\rho(t)$, the exponential convergence of $\rho(t)$ towards $\rho_{M_{0}}$ in $L^{1}(\mathbb{R})$ or $L^{2}(\mathbb{R})$ norms can be proved in a similar as in Section 3.4 .

\section{Conclusion and generalisations}

In this paper, the long time behaviours of the solutions to the nonlocal porous medium equation are studied, by showing the convergence of the relative entropy between the solutions and their Barenblatt profiles. The convergence in other norms can also be obtained, by assuming additional Hölder regularity on the solutions. Although we only concentrated on equations with power-law type absorption or convection, the same procedures can be applied to a larger class of models. For the general one-dimensional equation

$$
\rho_{t}-\nabla \cdot\left(\rho \nabla(-\Delta)^{-s} \rho+x \rho\right)=-P(t)^{-\delta} g(t, x, \rho)
$$

with absorption $g(t, x, \rho) \geq 0$, formally we have

$$
\begin{aligned}
\frac{\mathrm{d}}{\mathrm{d} t} H\left[\rho(t) \mid \rho_{M(t)}\right] & \leq-I[\rho(t)]-P(t)^{-\delta} \int(-\Delta)^{-s}\left(\rho(t)-\rho_{M(t)}\right) g(t, x, \rho) \mathrm{d} x \\
& \leq-2 H\left[\rho(t) \mid \rho_{M(t)}\right]+P(t)^{-\delta}\left\|(-\Delta)^{-s / 2}\left(\rho(t)-\rho_{M(t)}\right)\right\|_{2}\left\|(-\Delta)^{-s / 2} g(t, \cdot, \rho)\right\|_{2} .
\end{aligned}
$$

Similarly, for the equation

$$
\rho_{t}-\nabla \cdot\left(\rho \nabla(-\Delta)^{-s} \rho+x \rho\right)=-P(t)^{-\theta} \frac{\partial}{\partial x}\left(\rho^{1 / 2} h(t, x, \rho)\right),
$$


with general convection $\rho^{1 / 2} h(t, x, \rho)$ we obtain

$$
\frac{\mathrm{d}}{\mathrm{d} t} H\left[\rho(t) \mid \rho_{M_{0}}\right] \leq-I[\rho(t)]+P(t)^{-\theta}\|h(t, \cdot, \rho)\|_{2} I[\rho(t)]^{1 / 2} .
$$

As long as the solution $\rho$, the absorption $g$ or the convection $\rho^{1 / 2} h$ satisfy appropriate bounds, the exponential convergence of the relative entropy can also be established.

In addition to the above generalisations with other absorption or convection terms, many related problems are still widely open. The convergence results presented here can not be extended into higher dimensions, precisely because the entropy-entropy dissipation inequality is only proved in one dimension. Therefore, the clarification of this critical inequality in higher dimensions will shed lights on the behaviours of solutions to other associated equations. In the parameter regimes we considered in this paper, the absorption or convection eventually becomes exponentially small in the transformed equation with the similarity variables. In the other parameter regimes, the strength of the absorption or the convection becomes comparable to the nonlocal diffusion, usually leading to new nontrivial equations whose quantitative properties are much more difficult to study.

Acknowledgements We thank Prof. Juan Luis Vázquez for fruitful discussions concerning absorptiondiffusion and convection-diffusion models along with some regularity problems connected to model (2). We thank Felix Del Teso for useful discussions about the one dimensional integrated model obtained from (2) and related interesting numerical aspects. This work has been partially supported by GNAMPA of the Italian INdAM (National Institute of High Mathematics) and "Programma triennale della Ricerca dell'Università degli Studi di Napoli "Parthenope" - Sostegno alla ricerca individuale 2015-2017".

\section{References}

[1] Anton Arnold, Peter Markowich, Giuseppe Toscani, and Andreas Unterreiter. On convex Sobolev inequalities and the rate of convergence to equilibrium for Fokker-Planck type equations. Comm. Partial Differential Equations, 26(1-2):43-100, 2001.

[2] D. Bakry and Michel Émery. Diffusions hypercontractives. In Séminaire de probabilités, XIX, 1983/84, volume 1123 of Lecture Notes in Math., pages 177-206. Springer, Berlin, 1985.

[3] P. Biler, C. Imbert, and G. Karch. The nonlocal porous medium equation: Barenblatt profiles and other weak solutions. Arch. Ration. Mech. Anal., 215(2):497-529, 2015.

[4] Piotr Biler, Grzegorz Karch, and Régis Monneau. Nonlinear diffusion of dislocation density and self-similar solutions. Comm. Math. Phys., 294(1):145-168, 2010.

[5] A. Blanchet, V. Calvez, and J. A. Carrillo. Convergence of the mass-transport steepest descent scheme for the subcritical patlak-keller-segel model. SIAM Journal on Numerical Analysis, 46, 01 2008.

[6] A. Blanchet, J. Dolbeault, and B. Perthame. Two-dimensional Keller-Segel model: optimal critical mass and qualitative properties of the solutions. Electron. J. Differential Equations, pages No. 44, $32,2006$.

[7] L. Caffarelli, F. Soria, and J. L. Vázquez. Regularity of solutions of the fractional porous medium flow. J. Eur. Math. Soc. (JEMS), 15(5):1701-1746, 2013.

[8] L. A. Caffarelli and P. R. Stinga. Fractional elliptic equations, Caccioppoli estimates and regularity. Ann. Inst. H. Poincaré Anal. Non Linéaire, 33(3):767-807, 2016. 
[9] L. A. Caffarelli and J. L. Vázquez. Asymptotic behaviour of a porous medium equation with fractional diffusion. Discrete Contin. Dyn. Syst., 29(4):1393-1404, 2011.

[10] L. A. Caffarelli and J. L. Vázquez. Nonlinear porous medium flow with fractional potential pressure. Arch. Ration. Mech. Anal., 202(2):537-565, 2011.

[11] J. A. Carrillo, Y. Huang, M. C. Santos, and J. L. Vázquez. Exponential convergence towards stationary states for the 1D porous medium equation with fractional pressure. J. Differential Equations, 258(3):736-763, 2015.

[12] J. A. Carrillo and G. Toscani. Asymptotic $L^{1}$-decay of solutions of the porous medium equation to self-similarity. Indiana Univ. Math. J., 49(1):113-142, 2000.

[13] J. A. Carrillo and G. Toscani. Contractive probability metrics and asymptotic behavior of dissipative kinetic equations. Riv. Mat. Univ. Parma (7), 6:75-198, 2007.

[14] José A. Carrillo, Franca Hoffmann, Edoardo Mainini, and Bruno Volzone. Ground states in the diffusion-dominated regime. Calc. Var. Partial Differential Equations, 57(5):Art. 127, 28, 2018.

[15] D. Chafaï, N. Gozlan, and P. Zitt. First-order global asymptotics for confined particles with singular pair repulsion. Ann. Appl. Probab., 24(6):2371-2413, 2014.

[16] E. Chasseigne and E. R. Jakobsen. On nonlocal quasilinear equations and their local limits. $J$. Differential Equations, 262(6):3759-3804, 2017.

[17] A. de Pablo, F. Quirós, A. Rodríguez, and J. L. Vázquez. A general fractional porous medium equation. Comm. Pure Appl. Math., 65(9):1242-1284, 2012.

[18] F. Del Teso and E. R. Jakobsen. A convergent numerical method for the porous medium equation with fractional pressure. In preparation.

[19] E. Di Nezza, G. Palatucci, and E. Valdinoci. Hitchhiker's guide to the fractional Sobolev spaces. Bull. Sci. Math., 136(5):521-573, 2012.

[20] Jean Dolbeault and An Zhang. Flows and functional inequalities for fractional operators. Applicable Analysis, 96(9):1547-1560, 2017.

[21] A. C. Eringen. Nonlocal continuum field theories. Springer-Verlag, New York, 2002.

[22] M. Escobedo and O. Kavian. Asymptotic behaviour of positive solutions of a nonlinear heat equation. Houston J. Math., 14(1):39-50, 1988.

[23] M. Escobedo and E. Zuazua. Large time behavior for convection-diffusion equations in $\mathbf{R}^{N}$. J. Funct. Anal., 100(1):119-161, 1991.

[24] A. Gmira and L. Véron. Large time behaviour of the solutions of a semilinear parabolic equation in $\mathbf{R}^{N}$. J. Differential Equations, 53(2):258-276, 1984.

[25] A. Jüngel. Entropy methods for diffusive partial differential equations. SpringerBriefs in Mathematics. Springer, [Cham], 2016.

[26] S. Kamin and L. A. Peletier. Large time behaviour of solutions of the porous media equation with absorption. Israel J. Math., 55(2):129-146, 1986.

[27] S. Kamin and L. A. Peletier. Large time behaviour of solutions of the porous media equation with absorption. Israel J. Math., 55(2):129-146, 1986. 
[28] Ph. Laurençot and F. Simondon. Source-type solutions to porous medium equations with convection. Commun. Appl. Anal., 1(4):489-502, 1997.

[29] V. A. Liskevich and Yu. A. Semenov. Some problems on Markov semigroups. In Schrödinger operators, Markov semigroups, wavelet analysis, operator algebras, volume 11 of Math. Top., pages 163-217. Akademie Verlag, Berlin, 1996.

[30] R. J. McCann. A convexity principle for interacting gases. Adv. Math., 128(1):153-179, 1997.

[31] L. A. Peletier and D. Terman. A very singular solution of the porous media equation with absorption. J. Differential Equations, 65(3):396-410, 1986.

[32] L. A. Peletier and J. N. Zhao. Large time behaviour of solutions of the porous media equation with absorption: the fast diffusion case. Nonlinear Anal., 17(10):991-1009, 1991.

[33] G. Reyes and J. L. Vazquez. Asymptotic behaviour of a generalized Burgers' equation. J. Math. Pures Appl. (9), 78(6):633-666, 1999.

[34] X. Ros-Oton and J. Serra. Regularity theory for general stable operators. J. Differential Equations, 260(12):8675-8715, 2016.

[35] F. Santambrogio. Optimal transport for applied mathematicians, volume 87 of Progress in Nonlinear Differential Equations and their Applications. Birkhäuser/Springer, Cham, 2015. Calculus of variations, PDEs, and modeling.

[36] D. Stan, F. del Teso, and J. L. Vázquez. Existence of weak solutions for a general porous medium equation with nonlocal pressure. arXiv preprint arXiv:1609.05139, 2016.

[37] D. Stan, F. del Teso, and J. L. Vázquez. Finite and infinite speed of propagation for porous medium equations with nonlocal pressure. J. Differential Equations, 260(2):1154-1199, 2016.

[38] D. Stan, F. del Teso, and J. L. Vázquez. Porous medium equation with nonlocal pressure. arXiv preprint arXiv:1801.04244, 2018.

[39] G. Toscani. Entropy production and the rate of convergence to equilibrium for the Fokker-Planck equation. Quart. Appl. Math., 57(3):521-541, 1999.

[40] J. L. Vázquez. The porous medium equation. Oxford Mathematical Monographs. The Clarendon Press, Oxford University Press, Oxford, 2007. Mathematical theory.

[41] J. L. Vázquez and B. Volzone. Optimal estimates for fractional fast diffusion equations. J. Math. Pures Appl. (9), 103(2):535-556, 2015.

[42] C. Villani. Topics in optimal transportation, volume 58 of Graduate Studies in Mathematics. American Mathematical Society, Providence, RI, 2003. 